\title{
Effective Photodegradation of Methyl Orange Using Fluidized Bed Reactor Loaded with Cross-Linked Chitosan Embedded Nano-CdS Photocatalyst
}

\author{
Wai Szeto, ${ }^{1}$ Chi Wai Kan, ${ }^{1}$ C. W. M. Yuen, ${ }^{1}$ Shun-Wan Chan, ${ }^{2,3}$ and Kim Hung Lam ${ }^{2,3}$ \\ ${ }^{1}$ Institute of Textiles and Clothing, The Hong Kong Polytechnic University, Hung Hom, Hong Kong \\ ${ }^{2}$ Department of Applied Biology and Chemical Technology, The Hong Kong Polytechnic University, Hung Hom, Hong Kong \\ ${ }^{3}$ State Key Laboratory of Chirosciences, State Key Laboratory of Chinese Medicine and Molecular Pharmacology, Shenzhen, China \\ Correspondence should be addressed to Shun-Wan Chan; bcswchan@polyu.edu.hk and Kim Hung Lam; bcjoelam@polyu.edu.hk
}

Received 12 February 2014; Accepted 29 June 2014; Published 5 August 2014

Academic Editor: Dmitry Murzin

Copyright ( 2014 Wai Szeto et al. This is an open access article distributed under the Creative Commons Attribution License, which permits unrestricted use, distribution, and reproduction in any medium, provided the original work is properly cited.

\begin{abstract}
Chitosan-based photocatalyst composites containing CdS nanocrystals with and without glutaraldehyde or epichlorohydrin crosslinking treatments were investigated and the catalyzed photodegradation of methyl orange in aqueous solution was examined. In addition, the effects of catalyst dosage, initial dye concentration, and initial $\mathrm{pH}$ of the dye solution on the photodegradation kinetics were investigated. In this study, the effect of initial solution $\mathrm{pH}$ was more important than other factors. The photocatalyst composite could remove $99 \%$ dye in 80 minutes at $\mathrm{pH}$ 4. The catalyst composite was characterized by using X-ray diffraction (XRD), transmission electron microscopy (TEM), thermogravimetric analysis (TGA), fourier transform infrared (FT-IR), and visible reflectance spectroscopy. The dye removal mechanism of methyl orange involved an initial sorption process followed by photodegradation. The sorption process underwent the pseudo-second order kinetics, while photodegradation followed the Langmuir-Hinshelwood kinetics. Although the glutaraldehyde cross-linked chitosan enhanced the initial dye sorption, the epichlorohydrin cross-linked catalyst composite demonstrated a better overall dye removal performance, especially in the photodegradation step. Both chitosan encapsulated catalyst with and without epichlorohydrin cross-linking demonstrated the same pseudo-first order photodegradation kinetic constant of $0.026 \mathrm{~min}^{-1}$ and the same dye removal capacity. The catalyst composite could be reused but the photocatalytic activity dropped successively in each cycle.
\end{abstract}

\section{Introduction}

The textile industry is a major source of textile wastewater pollution [1]. Wastewater from textile plants often contains pollutants with intense color and requires treatment before discharge. These pollutants are potential threats to the environment, and new technology to remove dyes from wastewater is highly desired [2]. Due to the high variability of the compositions of textile wastewater and the increasing stability of dye stuff against biodegradation, most conventional physicochemical methods such as flocculation, adsorption, and biological treatment are relatively ineffective for the removal of dye stuff $[3,4]$. Moreover, biodegradation requires nutrients $[5,6]$ and the resulting products could be more toxic than the original contaminants [7]. Recently, much attention has been paid to catalyzed photodegradation that makes use of semiconductor photocatalysts, where photoexcitation of semiconductor generates holes and electrons that may act as oxidizing agent and reducing agent and trigger various degradation reactions of dyes [4, 8-10]. Most researchers are concerned about the strength of the semiconductor catalysts, which are usually produced in the form of nanosized particles. However, posttreatment separation of nanocatalysts is difficult with high costs and time consuming [11-18] and discourages their recovery. Moreover, nanocatalysts are prone to aggregate and reduce catalytic efficiency as the aggregation impedes the transfer of light-generated charge carriers to surface and increase the probability of recombination $[3,13$, 15, 18-20]. Furthermore, some semiconductors, such as $\mathrm{CuO}$ [20] and CdS [14, 21], are vulnerable to photocorrosion and 
would release heavy metals that are secondary pollutants. Alginate [11, 22], cellulose acetate [12], chitosan [3, 4, 19, 2326], and poly(diallyl dimethylammonium) chloride [21] were reported examples of immobilization materials for stabilizing the photocatalysts and improving their practical usability as well as their recollection and reusability after wastewater treatment $[25,27,28]$. In order to achieve high removal efficiency, the immobilization materials should allow adsorption and penetration of contaminants for photodegradation to happen. The chitosan can bring more dye molecules into close proximity via adsorption and the catalytic sites of the semiconductor react with them [25]. The adsorbed dyes can function as photosensitizers and the excited dye molecules inject high energy electron into conduction band of semiconductor and this phenomenon provided an alternative route for degradation [4]. Although chitosan is a common water-soluble biopolymer, it is fragile and modification is usually required to improve its mechanical strength for application. In particular, chemical cross-linking, such as glutaraldehyde [3, 26, 28, 29], epichlorohydrin [16, 27], and tripolyphosphate [27], were reported to improve strength and for the immobilization of photocatalysts.

In this work, chitosan with immobilzed CdS nanoparticles were prepared and the effects of degradation of the methyl orange (Figure 1) under various experimental conditions were investigated. The cross-linked and noncrosslinked chitosan matrices were studied. Other factors such as $\mathrm{pH}$ effect and initial dye concentration would also be investigated and the reusability of the photocatalyst was examined.

As fluidized bed reactor allows excellent contact between catalysts and reactants as well as good heat and mass transfer [30-32], a fluidized bed photoreactor with an immersed lamp tube was adopted in this work.

\section{Experimental}

2.1. Materials. All chemicals were obtained from SigmaAldrich unless otherwise specified. The purchased commercially available chitosan was originated from shrimp shells. Cadmium nitrate $\left(\mathrm{Cd}\left(\mathrm{NO}_{3}\right)_{2} \cdot 4 \mathrm{H}_{2} \mathrm{O}\right)$ and thiourea $\left(\left(\mathrm{NH}_{2}\right)_{2} \mathrm{CS}\right)$ were used as the precursors for the preparation of CdS. $50 \%(\mathrm{w} / \mathrm{w})$ glutaraldehyde in water (purchased from Acros) and epichlorohydrin were used as the cross-linking agents. Methyl orange (MO) was obtained as commercial products from International Laboratory USA and selected as the dye for our photochemical degradation studies without further purification. Milli-Q water was used for solution preparation and $A R$ grade sodium hydroxide $(\mathrm{NaOH})$ and nitric acid $\left(\mathrm{HNO}_{3}\right)$ were employed for $\mathrm{pH}$ adjustment.

\subsection{Preparation of Various Chitosan Based Catalyst Compos-} ites. The chitosan-based catalyst composites were prepared according to Zhu and coworkers [3, 28, 29]. The stock chitosan solution $(2 \% \mathrm{w} / \mathrm{w})$ was prepared by dissolving commercially available chitosan into $1 \% \mathrm{w} / \mathrm{w} \mathrm{HNO}_{3}$, and the solution was centrifuged to remove nondissolvable matter. $100 \mathrm{~mL}$ of the stock chitosan solution was mixed with $3.07 \mathrm{~g}$ of $\mathrm{Cd}\left(\mathrm{NO}_{3}\right)_{2} \cdot 4 \mathrm{H}_{2} \mathrm{O}$ solution $(62 \%$ w/w $)$ overnight

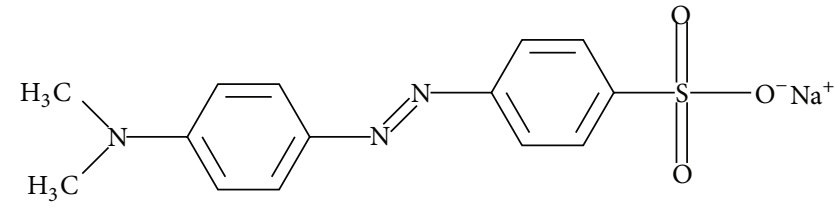

FIGURE 1: Chemical structure of methyl orange.

using a magnetic stirrer. Then, $300 \mathrm{~mL}$ of DDI water was added before the dropwise addition of $60 \mathrm{~mL}$ of $1 \mathrm{M} \mathrm{NaOH}$. Further stirring and sonication allowed solidification of all chitosan. The liquor was then discarded and the chitosan was homogenized into smaller pieces. Milli-Q water was added and the $\mathrm{pH}$ of the resultant mixture was adjusted to 10 by adding $10 \% \mathrm{w} / \mathrm{w} \mathrm{HNO}_{3}$ solution under magnetic stirring. The mixture was heated to $60^{\circ} \mathrm{C}$, and $10 \mathrm{~mL}$ of the thiourea solution $(40 \mathrm{mg} / \mathrm{mL})$ was added dropwise within 45 minutes. $0.3 \mathrm{M} \mathrm{NaOH}$ was added to keep the reaction at $\mathrm{pH} 10$. After the addition of thiourea solution, the solution was stirred at $60^{\circ} \mathrm{C}$ for another $1 \mathrm{~h}$. The resultant solid was washed with Milli-Q water and then methanol twice before oven-drying at $50^{\circ} \mathrm{C}$. In this study, the effect of using various crosslinking agents was also investigated by using glutaraldehyde or epichlorohydrin. Cross-linked catalyst composites were prepared using a procedure similar to that described by Wan Ngah et al. [33]. For the preparation of glutaraldehyde crosslinked composite, $1.50 \mathrm{~g}$ of composite was stirred in $120 \mathrm{~mL}$ Milli-Q water. Then, $1.0 \mathrm{~mL} \mathrm{50 \%} \mathrm{w/w} \mathrm{glutaraldehyde} \mathrm{was}$ added and the $\mathrm{pH}$ of the solution mixture was adjusted to 8.0 by using $0.3 \mathrm{M} \mathrm{NaOH}$. The reaction mixture was stirred overnight. For the preparation of epichlorohydrin crosslinked composite, $3.00 \mathrm{~g}$ of composite in $300 \mathrm{~mL}$ of water at $50^{\circ} \mathrm{C}$ and the solution $\mathrm{pH}$ was adjusted to 10.5 by adding $0.3 \mathrm{M} \mathrm{NaOH}$ before $1.0 \mathrm{~mL}$ epichlorohydrin was added. The reaction mixture was then further stirred for another $3 \mathrm{~h}$. The resultant cross-linked catalyst composites were then washed with Milli-Q water and methanol twice before drying in an oven at $50^{\circ} \mathrm{C}$.

2.3. Characterization of Catalyst Composites. X-ray diffraction (XRD) patterns were taken to characterize crystal structures inside the samples on a Rigaku SmartLab X-ray diffractometer. The Bragg-Brentano focusing mode was used. Measurement was performed with $\mathrm{Cu} \mathrm{K} \alpha$ radiation $(\lambda=1.54 \AA)$ in the range of $2 \theta=10-80^{\circ}$. The scanning speed, tube voltage, and anode current were $5^{\circ} / \mathrm{min}, 45 \mathrm{kV}$, and $200 \mathrm{~mA}$, respectively. Transmission electron microscopy (TEM) images were obtained by using a JEOL JEM-2100 transmission electron microscope at acceleration voltage of $200 \mathrm{kV}$. Thermogravimetric analysis (TGA) of the samples was carried out with 2$4 \mathrm{mg}$ of samples on a PerkinElmer TGA7 thermogravimetric analyzer performed under nitrogen atmosphere from 30 to $780^{\circ} \mathrm{C}$ with a heating rate of $5^{\circ} \mathrm{C} / \mathrm{min}$. The Fourier transform infrared (FT-IR) spectra, with wave number between 4000 and $450 \mathrm{~cm}^{-1}$, were recorded using the $\mathrm{KBr}$ disk method on a PerkinElmer Spectrum100 FTIR spectrometer. Visible reflectance spectra were obtained using a Konica Minolta CM-3500d spectrophotometer. 


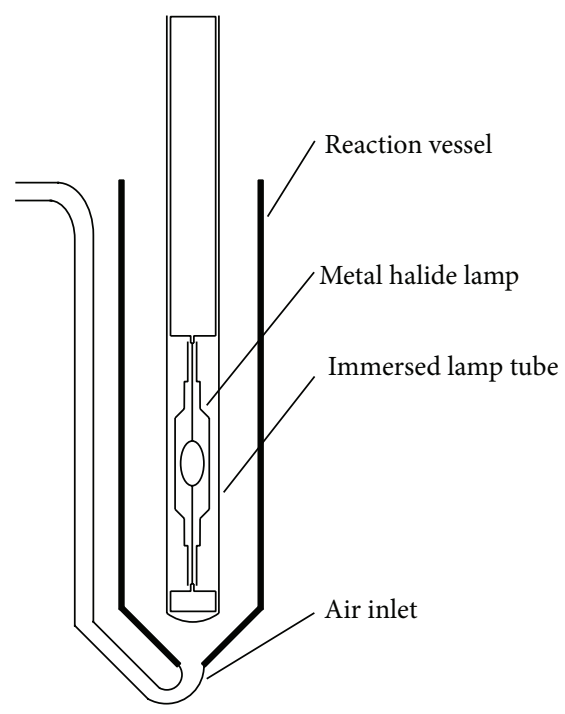

(a)

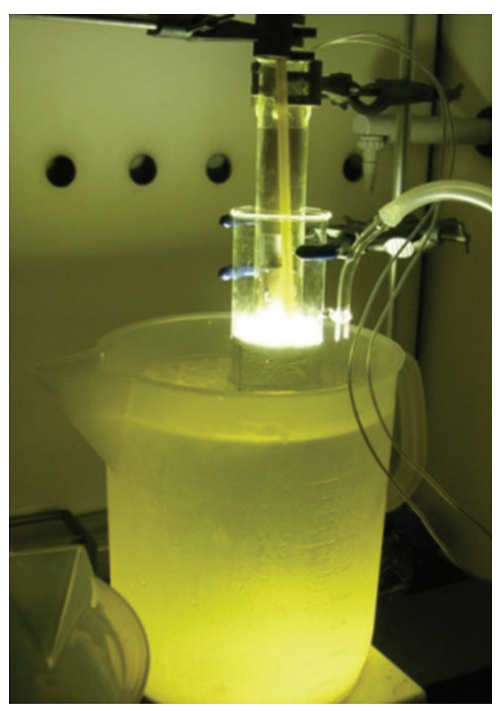

(b)

FIGURE 2: The photoreactor: (a) structure and (b) during photodegradation operation.

2.4. Adsorption and Photodegradation of Dye. Adsorption experiments were carried out before the photodegradation experiments. $0.3 \mathrm{~g}$ photocatalyst was stirred and mixed with $280 \mathrm{~mL}$ dye solution at a concentration of $10 \mathrm{ppm}$ unless otherwise specified. The adsorption kinetics were investigated by taking $2.0 \mathrm{~mL}$ of the sample solution at regular time intervals $(0,1,5,10,15,20,30$, and 60 minutes $)$ and monitored for 1 hour. In this study, pseudo-second order adsorption model was employed for studying color removal via adsorption of selected dyes. The linearized form of the kinetic model [34] (see (1)) is presented as follows:

$$
\frac{t}{Q_{t}}=\frac{1}{k_{2} Q_{e}^{2}}+\frac{t}{Q_{e}} .
$$

Adsorption capacity $\left(Q_{t}\right)$ can be determined from the concentration of dye solution by the expression

$$
Q_{t}=\frac{\left(C_{0}-C_{t}\right) V}{m},
$$

where $Q_{t}=$ adsorption capacity at time $=(t) ; Q_{e}=$ equilibrium adsorption capacity; $C_{0}=$ initial dye concentration; $C_{t}=$ dye concentration at time $=(t) ; V=$ volume of dye solution; $m=$ mass of adsorbent; $k_{2}=$ pseudo-second order adsorption rate constant; and $t=$ time for adsorption.

In order to study the photocatalytic degradation of MO, the mixture was transferred to the fluidized bed photoreactor [30] with a maximum volume of $350 \mathrm{~mL}$ (Figure 2). It consists of an immersed lamp tube with a $150 \mathrm{~W}$ UV blocked (broadband emission mainly at 400-600 nm with UV leakage < $26 \mathrm{~mW}$ ) metal halide lamp (OSRAM POWERSTAR HQITS $150 \mathrm{~W} / \mathrm{D})$, and a reaction vessel with an inverted conical bottom welded to a glass tube for air supply. Air supply could also function as agitation for the reaction. Before photodegradation, air was purged for $1 \mathrm{~h}$ without light illumination to allow adsorption equilibrium. The photodegradation reaction was conducted at $25^{\circ} \mathrm{C}$ using an ice water bath for cooling purpose. The photodegradation kinetics were investigated by taking $2.0 \mathrm{~mL}$ of the sample solution at appropriate time intervals $(0,5,10,15,20,25,30,45,60$, and 75 minutes unless otherwise specified). The obtained samples were centrifuged to remove any suspended photocatalyst and then analyzed by a PerkinElmer Lambda $25 \mathrm{UV}$ visible absorption spectrometer, and spectra with wavelength ranged from 210 to $700 \mathrm{~nm}$ were recorded and absorbance at $464 \mathrm{~nm}$ was monitored as a basis for determination of the $\mathrm{MO}$ concentration at various time intervals. The aqueous concentration of MO was monitored as described above where adsorption and photodegradation kinetics for catalyst composites with or without cross-linking with glutaraldehyde or epichlorohydrin were investigated. The effect of catalyst dosage, initial solution $\mathrm{pH}$, and initial dye concentration for the catalyst composite could be studied via the continuous monitoring of the dye concentration.

Pseudo-first order [3, 4, 9, 25, 26, 28, 35-38] (commonly known as Langmuir-Hinshelwood kinetic model [12]) kinetic model was employed for studying color removal via photodegradation of the selected dyes. The model equation (3) is presented as follows:

$$
\ln \frac{C_{0}}{C_{t}}=k_{1 p} t
$$

where $C_{t}$ is the concentration of dye at reaction time $=(t), C_{0}$ is the original concentration of dye at time $=0$, and $k_{1 p}$ is the pseudo-first order kinetic constant.

In order to ensure the color removal in the photodegradation step instead of adsorption, the amount of residual dye that had been adsorbed onto the photocatalyst composite after photodegradation was determined by (1) soaking the used photocatalysts into $40 \mathrm{~mL} 0.5 \mathrm{M} \mathrm{NaOH}$ solution and (2) measuring the concentration of dye extracted from the used photocatalysts. The residual dye present in the photocatalysts could be determined by analyzing the dye concentration of 
the resultant $\mathrm{NaOH}$ solution using a UV-visible absorption spectrometry, and the percentage of total residual dye is estimated with

$$
\frac{V_{\mathrm{des}} C_{\mathrm{des}}+V_{75} C_{75}}{V_{0} C_{0}} \times 100 \%,
$$

where $C_{0}=$ initial dye concentration; $C_{75}=$ dye concentration after photodegradation; $C_{\text {des }}$ is dye concentration after desorption; $V_{0}=$ initial volume of dye solution; $V_{75}=$ volume of dye solution after photodegradation; and $\mathrm{V}_{\mathrm{des}}=$ volume of aqueous $\mathrm{NaOH}$ used for the dye desorption.

\section{Results and Discussion}

3.1. XRD and TEM. Soltani and coworkers [39] reported the XRD patterns of cubic and hexagonal CdS nanocrystals. For the cubic nanocrystals, peaks could be observed at $2 \theta$ values of $26.4^{\circ}, 43.8^{\circ}, 51.9^{\circ}$, and $71.2^{\circ}$, which corresponded to the (111), (220), (311), and (331) crystalline planes of the face centered cubic structure, respectively. For the hexagonal structure, peaks at $2 \theta$ values of $24.8^{\circ}, 26.5^{\circ}, 28.2^{\circ}, 36.6^{\circ}, 43.7^{\circ}$, $47.8^{\circ}, 51.8^{\circ}, 67.1^{\circ}$, and $75.9^{\circ}$, which matched perfectly with the (100), (002), (101), (102), (110), (103), (112), (203), and (204) crystalline planes, respectively, were observed. Figure 3 shows the XRD analysis for the CdS crystal structures of the composite, and the result was similar to that reported by Zhu et al. [3]. Peaks were observed in the XRD pattern at $2 \theta$ values of $25.3^{\circ}, 26.8^{\circ}, 28.0^{\circ}, 43.9^{\circ}, 48.0^{\circ}, 52.2^{\circ}, 67.0^{\circ}$, and $74.8^{\circ}$. It is interesting to find out that the XRD pattern possesses identical peak positions but it has a different pattern of intensity than that of the hexagonal CdS structure as reported by Reyes and Velumani [40] as well as standard CdS (ICSD \#: 067776). A similar XRD pattern was reported by Maier et al. [41] and they reported that this observation was due to a mixture of crystals of the both forms. Overlapping the peaks with miller indices of (111), (220), and (311) of cubic $\mathrm{CdS}$ as well as the peaks with miller indices of (002), (110), and (112) of hexagonal CdS results in the XRD pattern shown. The peak at $2 \theta=19.8^{\circ}$ might be the crystalline peak of chitosan [42], and the broadness of XRD peaks indicated the existence of nanocrystals. The average size of the crystalline structures of CdS inside the composite was calculated by analyzing the XRD data of the peak at $2 \theta=43.9^{\circ}$ with the Scherrer's formula [41], and the size of the nanocrystal calculated was $46.0 \AA$.

Figure 4(a) is a TEM image that shows the catalyst composite particles containing CdS nanoparticles, the dark grains encapsulated in relatively transparent chitosan. Figure $4(\mathrm{~b})$ is the observation under higher power magnification, with the crystalline CdS nanoparticles encapsulated in the amorphous chitosan. The size of the CdS nanocrystal grains observed are comparable with the average size determined by XRD data using Scherrer's formula $(4.6 \mathrm{~nm})$. Extensive aggregation of CdS nanocrystals is observed in the TEM images (Figure 4(c)).

3.2. TGA. Thermal stability of the catalyst composite was assessed by thermal gravimetric analysis (Figure 5). The TGA curves showed 3 sections, $30-280^{\circ} \mathrm{C}, 280-400^{\circ} \mathrm{C}$, and $400-$ $780^{\circ} \mathrm{C}$. The first section showed about $12 \%$ of weight loss,

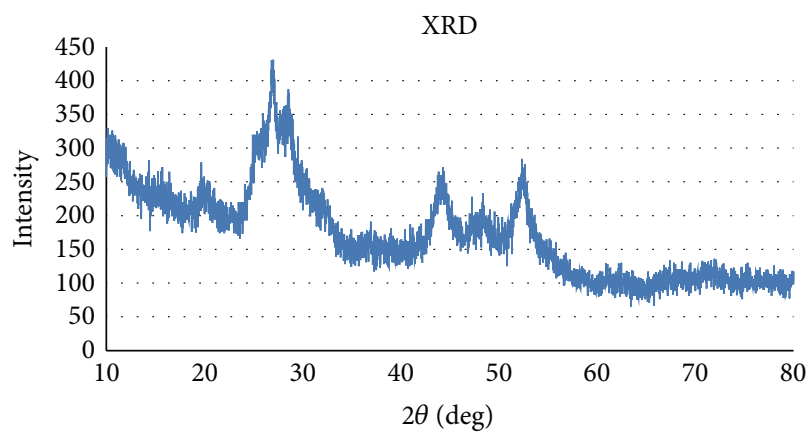

FIGURE 3: XRD spectra of the catalyst composite.

which should be the result of removal of adsorbed and bound water. The section $280-400^{\circ} \mathrm{C}$ demonstrated rapid weight loss and a specific decomposition reaction should be involved. The last section $400-780^{\circ} \mathrm{C}$ showed a relatively slow weight loss, which should be caused by some nonspecific decomposition reactions of the organic residuals.

3.3. FT-IR. Figure 6 shows the FT-IR spectra of (a) chitosan, (b) CdS-loaded chitosan without cross-linking, (c) epichlorohydrin cross-linked chitosan/catalyst composite, and (d) glutaraldehyde cross-linked chitosan/catalyst composite. The major difference between the pure chitosan and the CdSloaded chitosan was the shift of stretching vibration, which was at $3420 \mathrm{~cm}^{-1}$ for pure chitosan and $3397-3379 \mathrm{~cm}^{-1}$ after the introduction of CdS. In comparison with the noncross-linked composite (b), the peak associated with $-\mathrm{NH}_{2}$ bending, which was originally located at $1647 \mathrm{~cm}^{-1}$, shifted slightly to $1654 \mathrm{~cm}^{-1}$, indicating that the $-\mathrm{NH}_{2}$ might change to Schiff base $(\mathrm{C}=\mathrm{N})[43,44]$. The absorption band around $1562 \mathrm{~cm}^{-1}$ enhanced and formed a shoulder for glutaraldehyde cross-linked composite, which agreed with the observation by Monteiro and Airoldi [44]. This observation may be related to the self-reaction of glutaraldehyde to form unsaturated polyaldehydes via aldol condensation [45]. Hu and coworkers [46] also suggested that the observation might be due to the possibility that chitosan cross-linked by the unsaturated polyaldehydes. Another evidence shows that the cross-linking reaction can be reviewed by the weakened absorption peaks at 1420 and $1320 \mathrm{~cm}^{-1}$, where are the $\mathrm{NH}$ deformation vibration and $-\mathrm{CN}$ stretching vibration of free intact chitosan. Ngah and Fatinathan [47] suggested that the consumed amino groups after cross-linking reaction is a good explanation for two weakened infrared absorptions of chitosan. Further evidence shows that a small protrusion was found on the IR transmittance spectrum at $1105 \mathrm{~cm}^{-1}$ after the cross-linking step, and Beppu et al. [48] suggested that this might be due to the decrease in the number of aliphatic amino groups. The FTIR spectrum of the epichlorohydrin cross-linked catalyst composite was identical to the noncrosslinked one. Epichlorohydrin cross-linking consumed part of the $-\mathrm{OH}$ groups on chitosan back bone and formed new $\mathrm{C}-$ $\mathrm{O},-\mathrm{OH}$ groups and methylene groups and this change is not easily differentiated in the spectrum. 


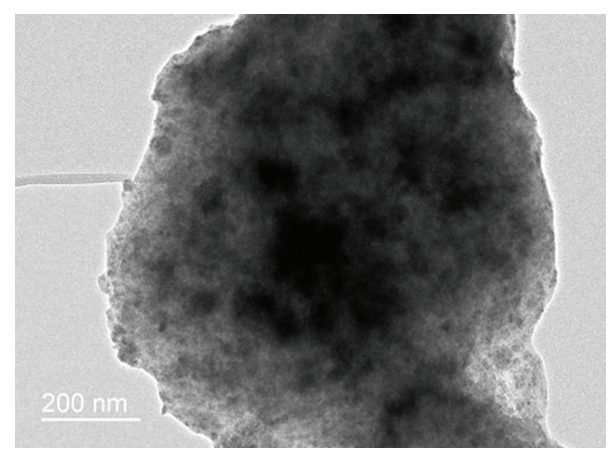

(a)

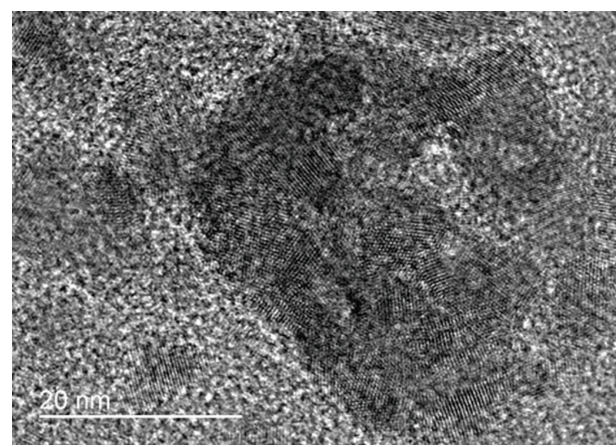

(b)

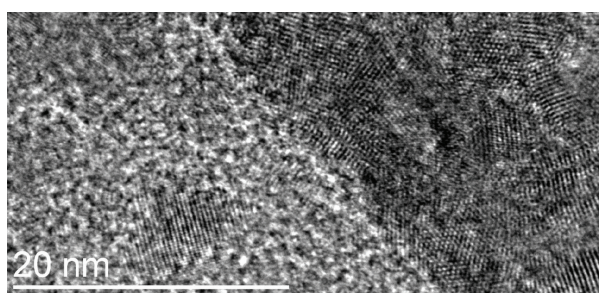

(c)

FIGURE 4: TEM images of catalyst composite (a) crystals encapsulated into relatively transparent chitosan, (b) agglomerated grains, and (c) CdS crystals.

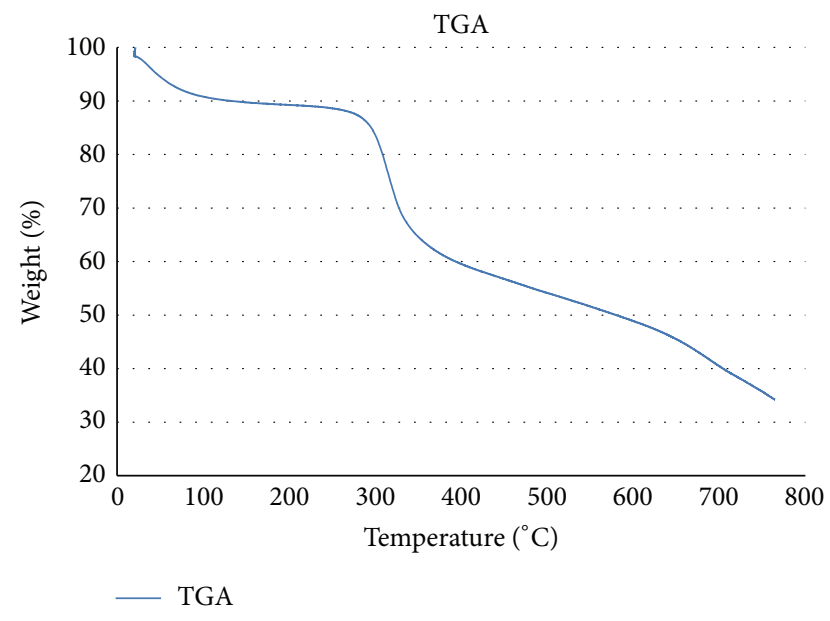

FIgURE 5: Thermal gravimetric curve of the catalyst composite.

3.4. Visible Reflectance. Band gap of semiconductor can be determined by measuring reflectance between $400 \mathrm{~nm}$ and $700 \mathrm{~nm}$ using the Tauc relation $[49,50]$. The band gap of certain semiconductor provides information on crystal size as increasing blue shift of absorption edge is observed with decreasing crystal size, caused by quantum confinement effect. According to Kumar and coworkers [49], the relation between optical absorption coefficient, wavelength of light, and band gap $\left(E_{g}\right)$ is as follows:

$$
\alpha h v=A\left(h v-E_{g}\right)^{1 / 2}
$$

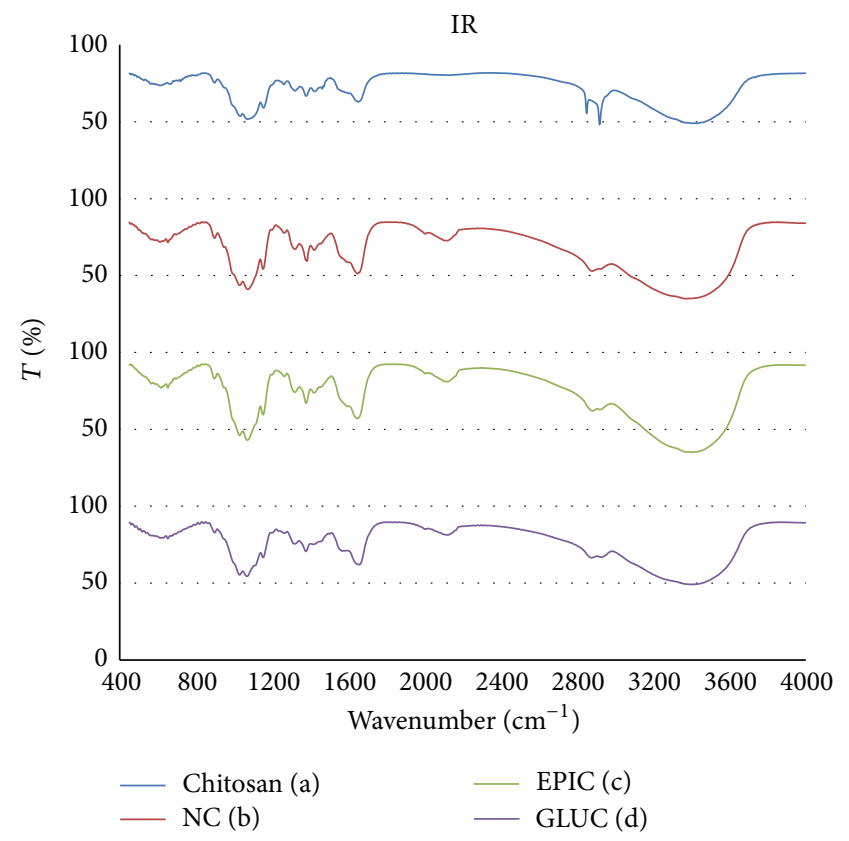

FIGURE 6: FT-IR spectra of (a) chitosan, (b) catalyst loaded chitosan without cross-linking, (c) epichlorohydrin cross-linked chitosan/catalyst composite, and (d) glutaraldehyde cross-linked chitosan/catalyst composite.

where $\alpha=$ absorption coefficient, $h v=$ light energy, $A$ is a constant that different for different transitions, and $E_{g}=$ band gap of material.

The Tauc plot of $(\alpha h v)^{2}$ against $h v$ yields a line, where the $x$-intercept of the straight line extrapolation gives the band 


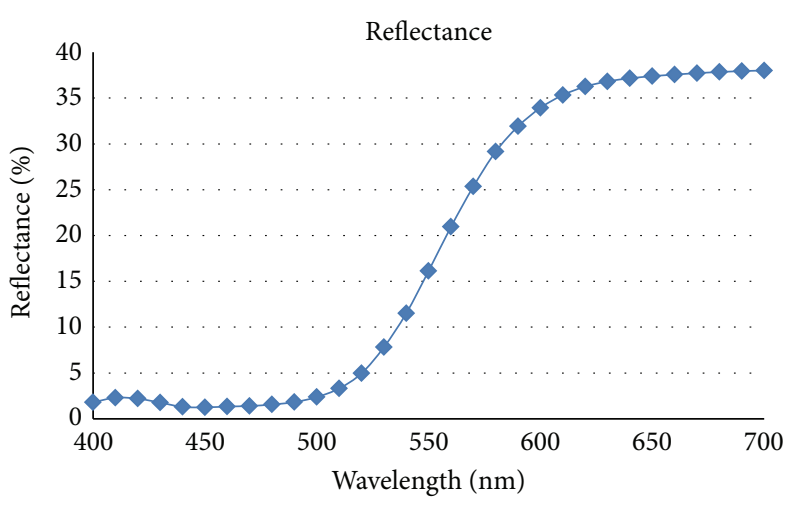

(a)

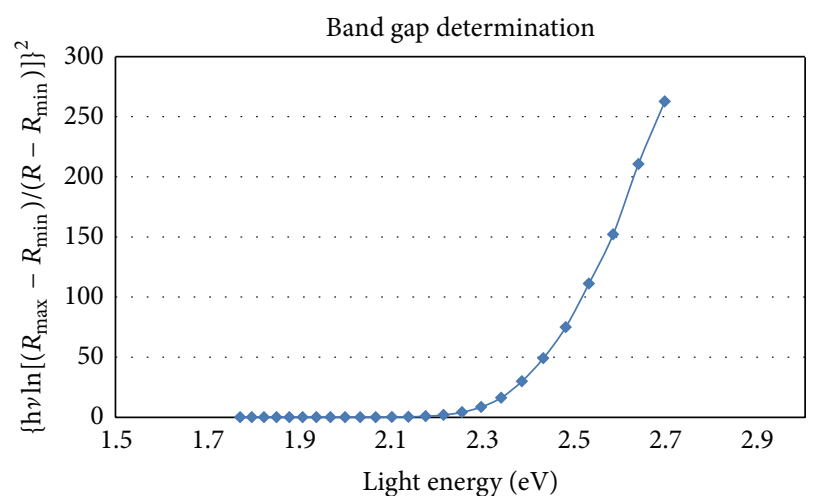

(b)

Figure 7: (a) \% reflectance against wavelength plot and (b) $\left\{h v \ln \left[\left(R_{\max }-R_{\min }\right) /\left(R-R_{\min }\right)\right]\right\}^{2}$ versus $h v$ plot.

gap energy $(h v)$. For opaque materials, it is difficult to measure the absorption coefficient and a mathematical model (see (6)) was used for the calculation of band gap energy $[49,50]$. Consider the following:

$$
\left\{h v \ln \left[\frac{R_{\max }-R_{\min }}{R-R_{\min }}\right]\right\}^{2} \text { versus } 2 h v,
$$

where $R_{\max }$ and $R_{\min }$ are the maximum and minimum values of reflectance, respectively, and $R$ is the reflectance at a given photon energy $h v$.

Figures 7(a) and 7(b) are the percentage of reflectance against wavelength plot and the corresponding Tauc plot, respectively. The determined $E_{g}$ of the composite is $2.40 \mathrm{ev}$. This value indicated that the band gap CdS inside the composite is identical to that of bulk CdS material [51].

3.5. Study of the Mechanism of Dye Degradation Using UVVisible Absorption Spectroscopy. The UV-visible spectra may provide information on the structural changes of dyes during the experiments. The whole process involved (i) an initial adsorption step with light off, (ii) a one-hour air purging step, and (iii) light on for photodegradation (Figure $8(\mathrm{~d})$ ). The air purging step is to ensure that the oxygen is not limited in the photodegradation step. Figures $8(a)$ and $8(d)$ depict changes in the UV-visible absorption spectrum and concentration changes throughout the adsorption and photodegradation steps. The characteristic of UV-visible absorption spectrum of MO solution is the 2 characteristic peaks; the main peak with maximum located at $464 \mathrm{~nm}$ that demonstrates color is arisen from whole chromophore with an azo group and another peak in the UV region with maximum located at $271 \mathrm{~nm}$ arisen from "benzene rings" structures of MO molecules [3]. During adsorption and air purging, both absorption peaks decreased with the same relative rate. After adsorption, for an hour, the MO concentration reduced to $70 \%$ of original value. The initial adsorption kinetics could be determined from the absorption peaks, and the kinetic data fitted the pseudo-second order rate law (Figure 8(b)). For the following photodegradation process, the two characteristic spectral absorption peaks (464 nm and $271 \mathrm{~nm}$ ) no longer decreased with the same relative rate (Figure $8(\mathrm{c})$ ) but with $k_{1 p}$ of 0.026 and $0.0063 \mathrm{~min}^{-1}$, respectively. The kinetic data implied that the catalytic photodegradation process was more efficient in breaking the azo bond than the aromatic rings. It is also interesting to observe that the peak originally located at $464 \mathrm{~nm}$ shifted towards $380 \mathrm{~nm}$ and the peak originally located at $271 \mathrm{~nm}$ shifted towards a lower wavelength throughout the photodegradation step. The shift of the peak originally within visible region would be caused by periphery group modifications of the dye [36] while that of the peak originally located at $271 \mathrm{~nm}$ would be caused by both destruction of the aromatic rings and overlapping with the growing absorption edge, located at wavelength $<240 \mathrm{~nm}$. The growth of the absorption edge was likely to be caused by simple fragments resulting from photodegradation. The system without photocatalyst (blank) showed no dye photolysis after 75 minutes of illumination (Figure 8(d)).

3.6. Effect of Cross-Linking of Catalyst Composite on Dye Removal. Researchers always crosslink chitosan to improve its mechanical strength, heat resistance [26], and chemical stability [16]. Natural chitosan dissolves readily in acidic $\mathrm{pH}$, and cross-linking could help its application at low $\mathrm{pH}$. Usually, cross-linking reactions are targeting the amino or hydroxyl groups of chitosan molecules. As reported, epichlorohydrin could cross-link with the hydroxyl groups while glutaraldehyde and tripolyphosphate could crosslink the amino groups [43, 44, 52]. Jawad and Nawi [16] reported that cross-linking by epichlorohydrin improved the catalytic activity of the $\mathrm{TiO}_{2} /$ chitosan film to result in a faster photodegradation of phenol; phenol removal can be improved from $58 \%$ to $95 \%$ within $2 \mathrm{~h}$, and the crosslinkage could enhance the chemical stability of the film against photodegradation. In contrast, Li et al. [27] found that degradation of methyl orange using $\mathrm{TiO}_{2}$ immobilized in tripolyphosphate cross-linked chitosan is much lower than that of its noncross-linked counterpart. Effect of crosslinking on dye removal was also analyzed in this work by comparing adsorption-photodegradation characteristics of 


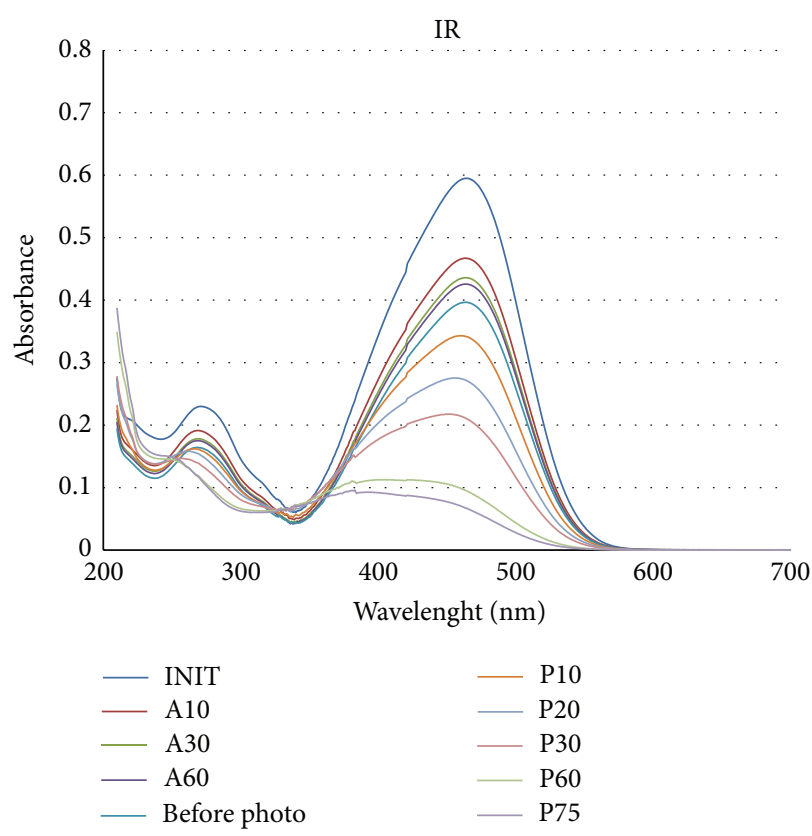

(a)

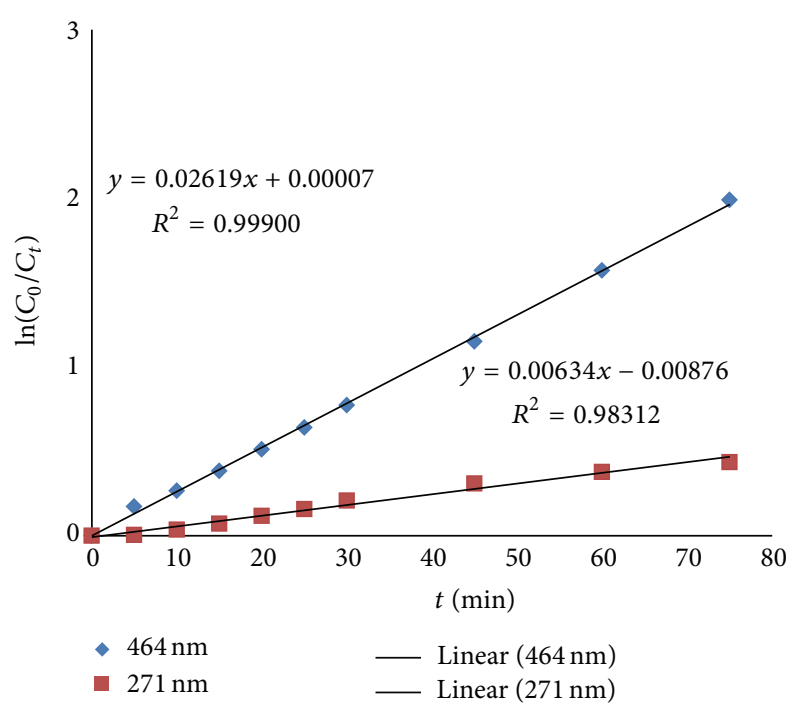

(c)
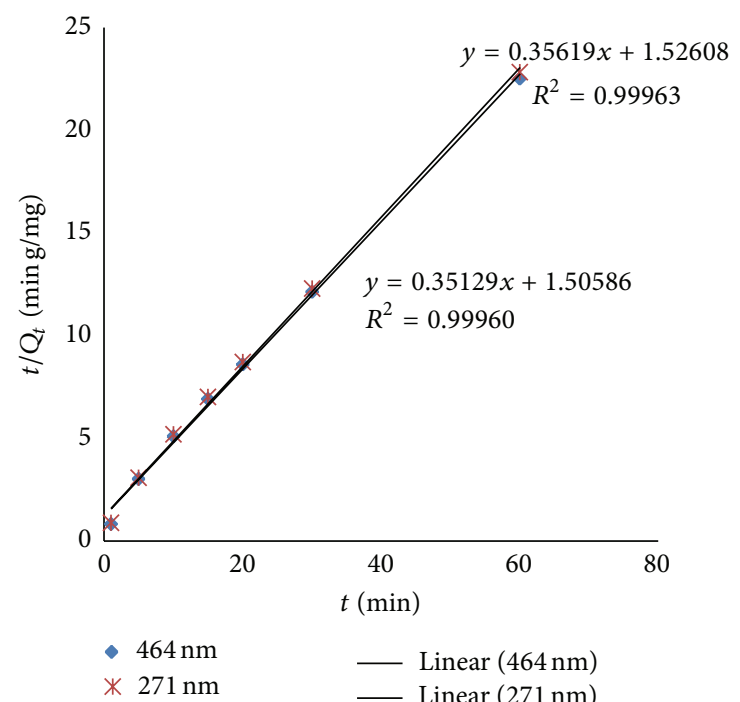

(b)

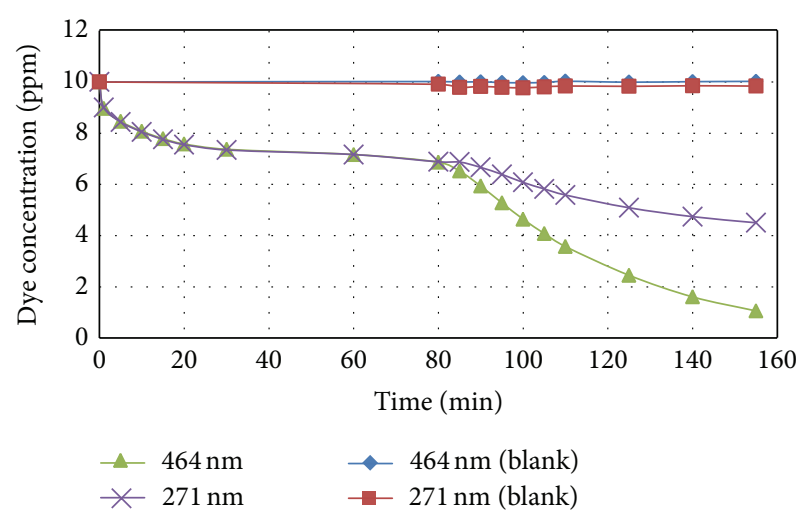

(d)

Figure 8: (a) UV-visible absorbance spectra of samples during experiment. (b) The corresponding linearized plots of pseudo-second order adsorption kinetics. (c) The corresponding linearized plots of pseudo-first order photodegradation kinetics. (d) The corresponding methyl orange adsorption-photodegradation curves.

noncross-linked and cross-linked catalyst composites. The CdS catalyst immobilized in chitosan was cross-linked using two cross-linking agents glutaraldehyde and epichlorohydrin while the noncross-linked chitosan immobilized catalyst (NC) was used as control. We found out that the glutaraldehyde cross-linked chitosan (GC) caused observable changes on both dye adsorption and photodegradation while the epichlorohydrin cross-linked chitosan (EC) showed similar removal efficiency of adsorption and photodegradation as compared with the noncross-linked control (NC) (Figure 9). The glutaraldehyde cross-linked chitosan (GC) remarkably enhanced the adsorption rate and capacity, implying that the amino groups on chitosan might be mainly responsible for dye adsorption and converting it into a Schiff base might alter and enhance the adsorption process [53]. A Schiff base would be more attractive than the original amino groups for these dye molecules bearing sulfonic acid groups. It is interesting to observe that the glutaraldehyde cross-linked chitosan embedded catalyst showed higher initial sorption than other samples. However, the final dye decolorization by the glutaraldehyde cross-linked composite was slightly inferior to the noncross-linked or epichlorohydrin cross-linked 


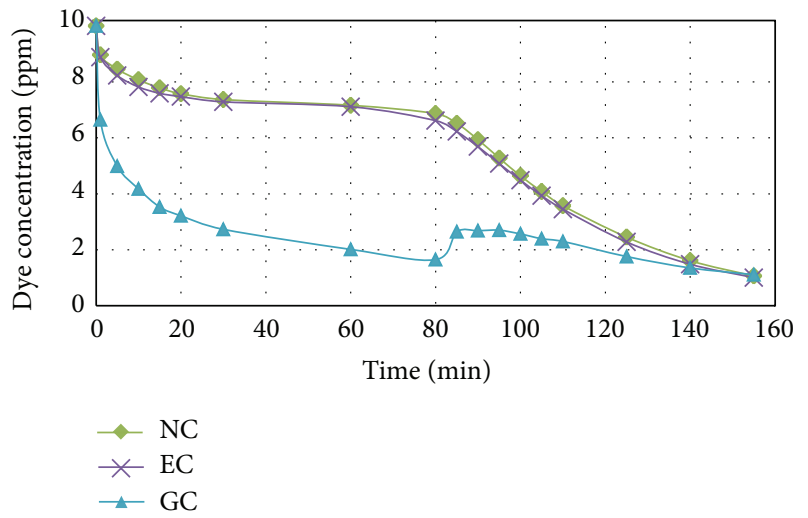

(a)

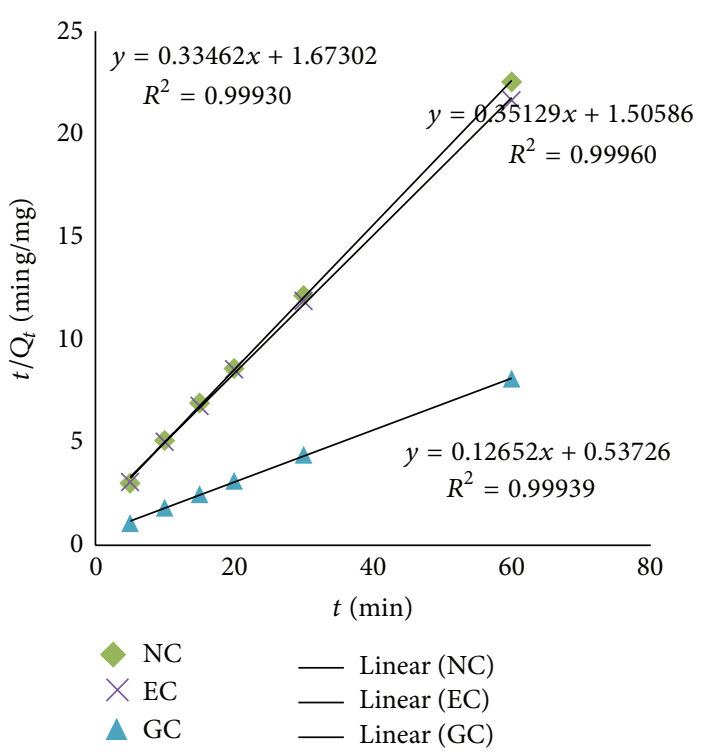

(b)

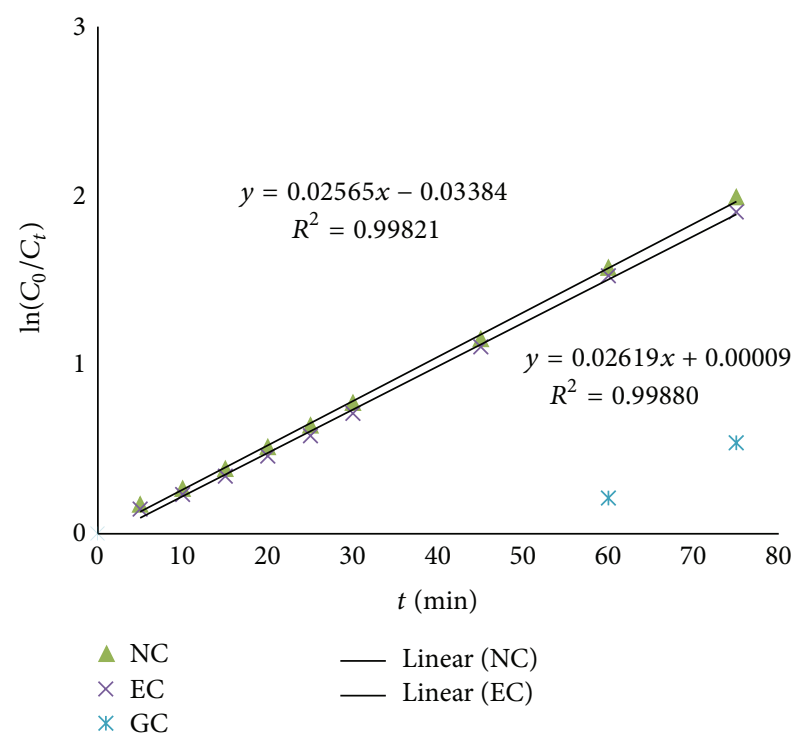

(c)

FIGURE 9: (a) Methyl orange adsorption-photodegradation curves (abbreviations: NC, EC, and GC) denote noncross-linked, epichlorohydrin cross-linked and glutaraldehyde, cross-linked chitosan catalyst composites, respectively. (b) The corresponding linearized plots of pseudosecond order adsorption kinetics. (c) The corresponding linearized plots of pseudo-first order photodegradation kinetics.

composite. For photodegradation using the glutaraldehyde cross-linked composite (GC), some adsorbed dye molecules were desorbed at the initial period of illumination, a phenomenon which might be due to the photodesorption of dye [54-58]. The pseudo-second order kinetic and the LangmuirHinshelwood kinetic models are commonly employed for comparing the adsorption and photodegradation kinetics using photocatalysts. In this work, we adopted these 2 models to compare the effectiveness of the catalysts, and the reaction kinetics can be expressed using kinetic plots for comparison. High correlation coefficients with $R^{2}>0.998$ were generally observed (Figure 9). It is interesting to point out that photodegradation using the glutaraldehyde crosslinked chitosan catalyst (GC) could not fit the LangmuirHinshelwood kinetic model $\left(R^{2}=0.65\right)$, and the reason might be accounted by the significant desorption at the initial period of illumination. The epichlorohydrin cross-linked chitosan catalyst (EC) showed similar rate constants of adsorption $\left(0.07 \mathrm{~g} / \mathrm{mg} \mathrm{min}\right.$.) and photodegradation $\left(0.026 \mathrm{~min}^{-1}\right)$ to the noncross-linked control (NC). Chitosan encapsulated $\mathrm{CdS}$ catalyst with and without epichlorohydrin cross-linking demonstrated the same final dye removal of $90 \%$. The glutaraldehyde cross-linking method enhanced adsorption and $80 \%$ of dye adsorbed in 1 hour while both the chitosan 


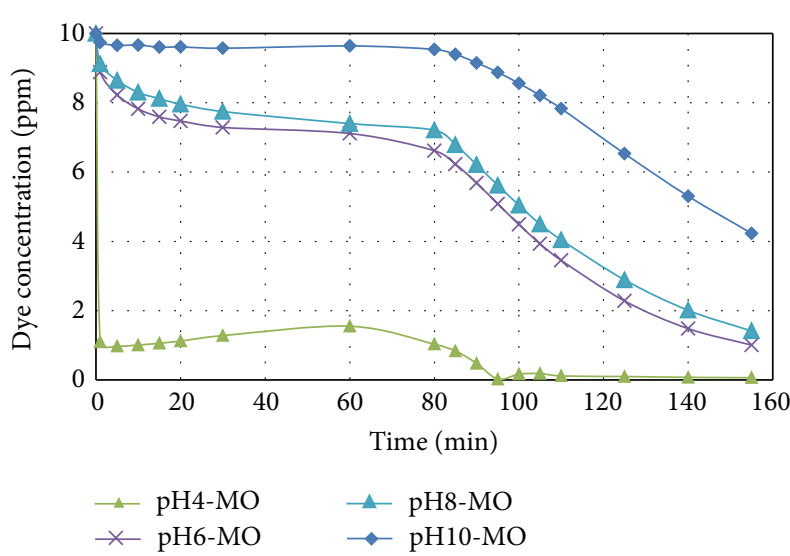

(a)

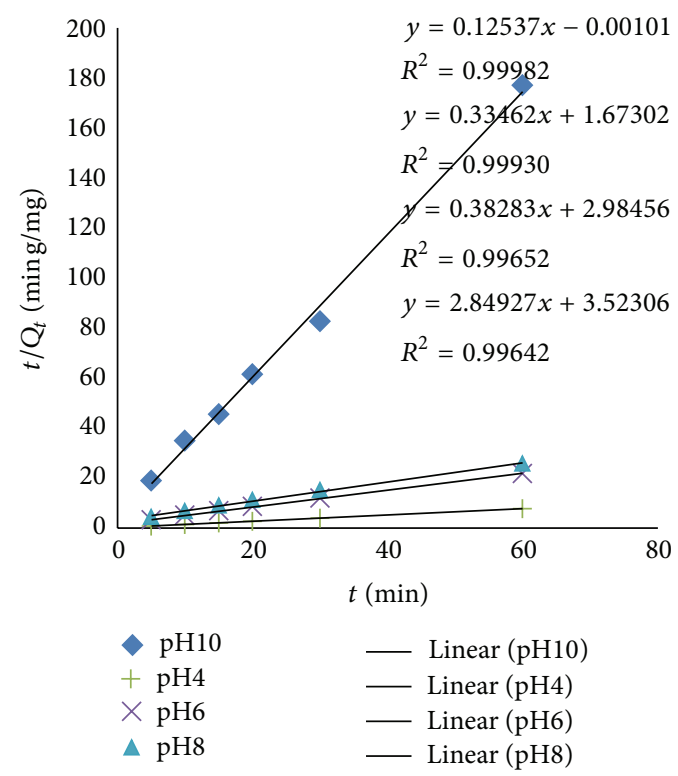

(b)

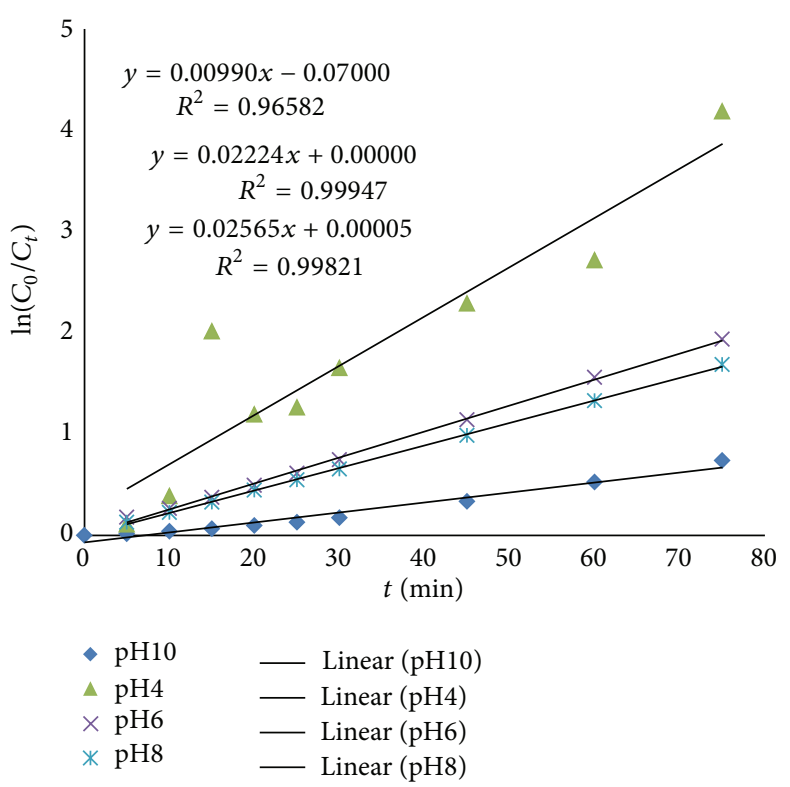

(c)

FIGURE 10: (a) Methyl orange adsorption-photodegradation by epichlorohydrin cross-linked catalyst composite at different initial solution $\mathrm{pH}$; (b) the corresponding linearized plots of pseudo-second order adsorption kinetics; and (c) the corresponding linearized plots of pseudofirst order photodegradation kinetics.

encapsulated CdS catalyst with and that without epichlorohydrin cross-linking could only adsorb $30 \%$ of the dye. However, the glutaraldehyde cross-linking method did not show any improvement in the photodegradation efficiency with the final dye removal of $89 \%$ and photodecolorization profile was distorted by photodesorption at the initial stage of the reaction. As we found out that glutaraldehyde crosslinked catalyst composite (GC) did not perform well as compared with epichlorohydrin cross-linked catalyst (EC), the remaining study would use the epichlorohydrin crosslinked catalyst composite only.
3.7. Effect of $\mathrm{pH}$ on Dye Removal. Solution $\mathrm{pH}$ affects the performance of photocatalysts significantly $[3,9,13,18,20$, $28,30,37,59-68]$, and the dye removal efficiency of the epichlorohydrin cross-linked catalyst composite at the initial $\mathrm{pH}$ values of $4,6,8$, and 10 was analyzed (Figure 10). The results showed that higher sorption rates and capacities occurred at lower solution $\mathrm{pH}$. As the point of zero charge of chitosan (around $\mathrm{pH} 8$ ), the amino groups would be protonated at a lower $\mathrm{pH}$ [69] while the sulfonic acid groups of methyl orange (MO) would remain ionized at the tested $\mathrm{pH}$ values. The strong electrostatic attraction 
between anionic MO molecules and cationic protonated amino groups of chitosan enhanced the adsorption at low $\mathrm{pH}[13,20,25]$. Figure 11 shows that photodegradation of MO which occurred in all tested $\mathrm{pH}$ values. The $\mathrm{MO}$ adsorption at $\mathrm{pH} 4$ was very high and the photodegradation at this $\mathrm{pH}$ was masked and hardly observed. For $\mathrm{pH} 6$ and 8, photodegradation removal of over $80 \%$ with 75 minutes of illumination except $\mathrm{pH} 10$ with photodegradation removal of over $50 \%$ only. The adsorption at $\mathrm{pH} 6,8$, and 10 followed the pseudo-second order kinetics well (Figure 10(b)) with high correlation coefficients $\left(R^{2}>0.996\right)$ and the rate determining step of adsorption seemed to be chemical adsorption in nature [70]. Figure 10(c) shows that the photodegradation process at $\mathrm{pH} 6,8$, and 10 followed the common LangmuirHinshelwood kinetics. At $\mathrm{pH} 4$, the high adsorption precluded the quantization of the dye photodegradation and the solution was too low for an accurate determination. In general, our experimental results agreed with Zhu et al's work [3]. In order to ensure the dye was photodegraded with the illumination instead of adsorption, the dye desorption was carried out after the experiment. Figure 11 depicts that the percentage of total residual dye after desorption was similar to the residual dye after the dye removal process (which included both the adsorption and photodegradation processes). The adsorption step could bring the dye molecules into close proximity to the catalytic sites of the semiconductor to react instantly with active species generated by the photocatalyst [20, 25]. Additionally, at $\mathrm{pH}$ below 4.4, the MO molecules exist in quinonoid form and the degradation rate could be further increased without the stable azo bond [13].

3.8. Dye Concentration on Dye Removal. Both the adsorption and photodegradation processes were found to be dye concentration-dependent $[3,8,26,28,37,59,65]$. Hence, experiments were carried out with different initial solution concentrations (5 to $20 \mathrm{ppm}$ ) (Figure 12(a)). Both the adsorption rate and capacity increased with initial dye concentration. Figure 12(b) indicates that the adsorption processes followed the pseudo-second kinetics order with high correlation coefficient $\left(R^{2}>0.9991\right)$ regardless of the initial dye concentration.

For photodegradation, a higher initial dye concentration would result in lower dye removal efficiency. The determined photodegradation kinetic data (Figure 12(c)) followed the pseudo-first order rate law and was comparable to that reported by Zhu et al. [3]. They reported that the rate constants for initial dye concentrations of 10 and $20 \mathrm{ppm}$ were $0.029 \mathrm{~min}^{-1}$ and $0.011 \mathrm{~min}^{-1}$, respectively, which are close to our results of $0.026 \mathrm{~min}^{-1}$ and $0.012 \mathrm{~min}^{-1}$, respectively. They proposed that the decreasing $k_{1 p}$ with the increasing dye concentration might be due to the high dye concentration which reduced the optical path length for light to reach the photocatalyst. Additionally, the increase in dye concentration will enhance the dye molecules adsorption on photocatalyst surface and prevent the adsorption of $\mathrm{OH}^{-}$and $\mathrm{O}_{2}$ for photodegradation. In general, we found out that the initial reaction rate increases with increasing dye concentration

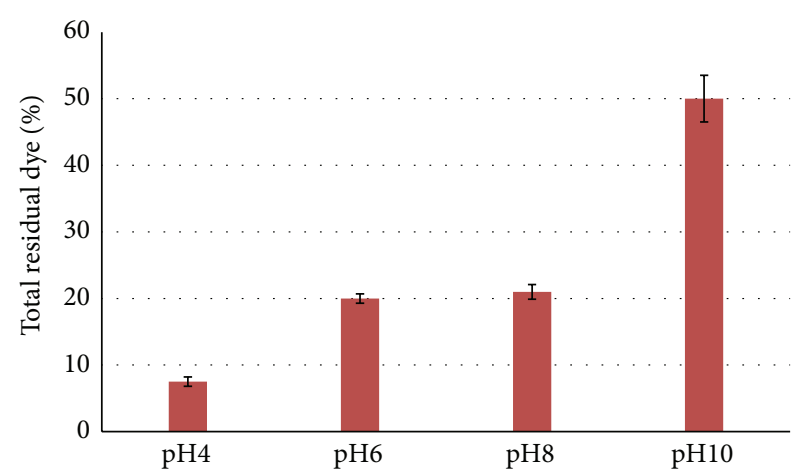

FIGURE 11: Percentage of residual dye after photodegradation at different initial solution $\mathrm{pH}$.

at relatively low solution concentration. This might be due to the reaction frequency between the active sites and the dye molecules could increase with dye concentration until an optimal value had been reached, and the photon absorption by dye molecule becomes critical at evaluated dye concentration [28]. The increased adsorbed interfering degradation intermediates [67] adsorbed onto active sites and reduced the adsorption of $\mathrm{OH}^{-}$and $\mathrm{O}_{2}$ essential for photodegradation.

3.9. Effect of Catalyst Dosage on Dye Removal. Photocatalyst dosage is one of the factors that determines the photodegradation kinetics $[9,37,59,65]$, and experiments were carried out in three dosages $(1.2,2.0$, and $2.8 \mathrm{~g} / \mathrm{L}$ ) (Figure 13). The results showed that both adsorption capacity and photodegradation rate increased remarkably with catalyst dosage. Increasing the catalyst dosage from 1.2 to $2.8 \mathrm{~g} /$ would increase the dye removal efficiency from $90 \%$ to $99 \%$ (Figure 13(a)). The adsorption process followed the pseudo-second order kinetics with increasing adsorption kinetic constant $\left(k_{2}\right)$ from 0.07 to 0.18 (Figure 13(b)) while the photodegradation kinetics followed the pseudo-first order kinetics with $k_{1 p}$ increasing from 0.026 to 0.06 (Figure 13(c)). It is obvious that the increase in catalyst dosage would increase the availability of active sites and more illuminated area for more dye molecules to be adsorbed and photodegraded $[9,28,37,65]$. Thus, the rate of photodegradation increased with the catalyst dosage.

3.10. Reusability of Catalyst Composites. Figure 14 shows the study of reusability of the catalyst composite. To evaluate changes in the efficiency and kinetic constants of reused photocatalyst, $2.8 \mathrm{~g} / \mathrm{L}$ of the catalyst was tested for 3 cycles. There were marginal drops in both the adsorption rate constant $\left(k_{2}\right)$ and equilibrium capacity $\left(Q_{e}\right)$ for the 3 cycles. The kinetic rate constant of photodegradation $\left(k_{1 p}\right)$ declined from 0.06 (1st cycle) to $0.03 \mathrm{~min}^{-1}$ (2nd cycle) and $0.023 \mathrm{~min}^{-1}$ (3rd cycle). The dye decolorization was mildly decreasing from $99 \%$ (1st cycle) to $94 \%$ in the 2nd cycle and $89 \%$ in the $3 \mathrm{rd}$ cycle. Generally, the catalyst remained sufficiently efficient after repeated use. The loss of activity of the photocatalyst 


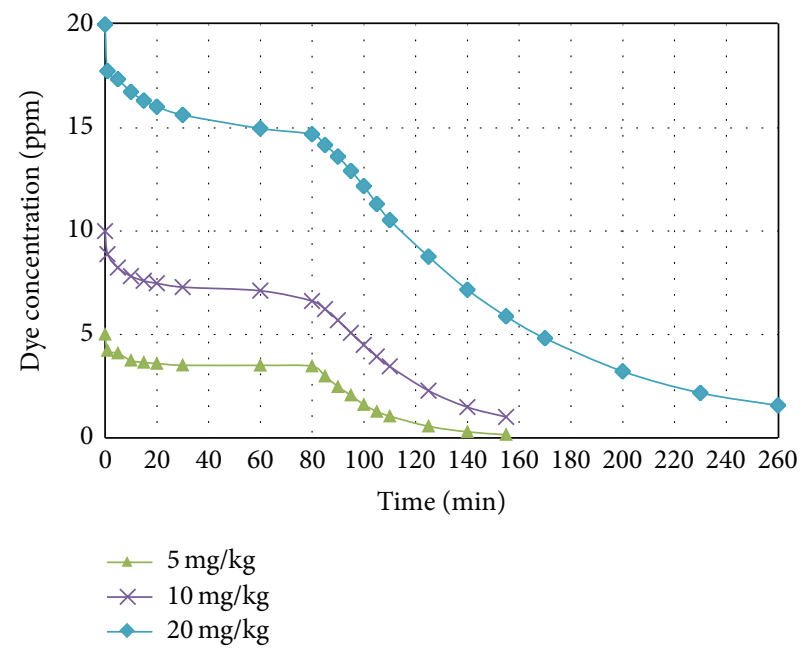

(a)

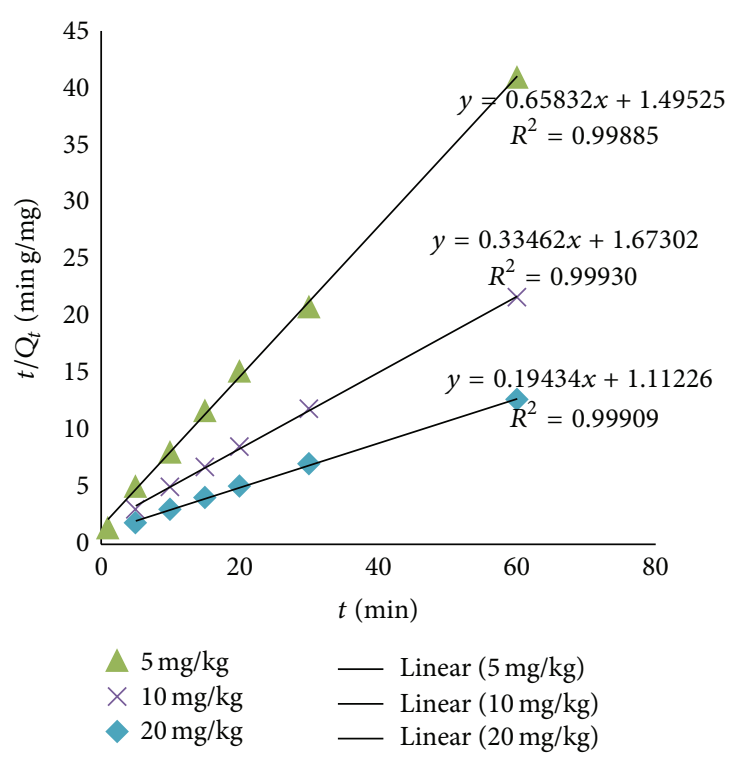

(b)

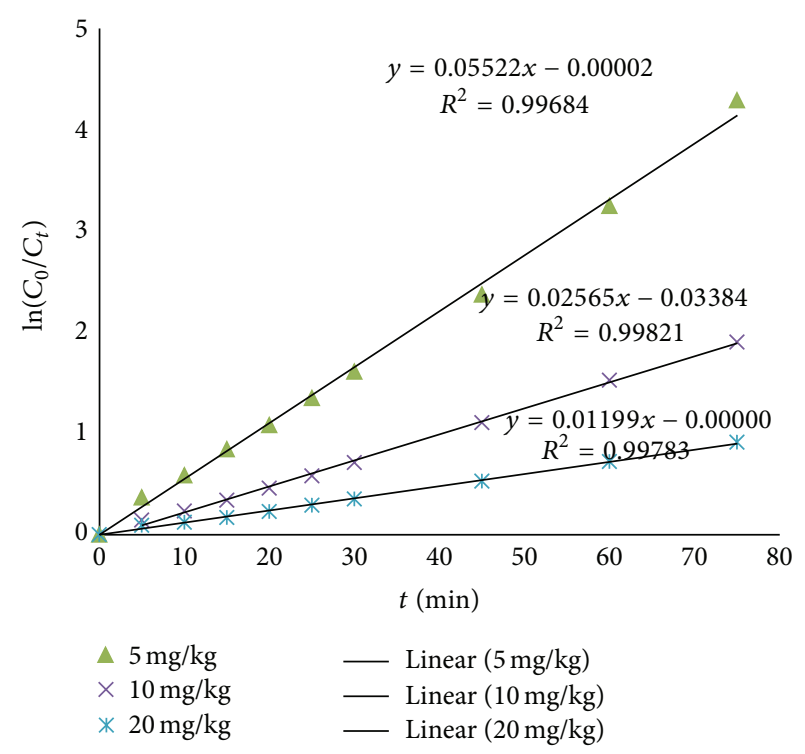

(c)

FIGURE 12: (a) Methyl orange adsorption-photodegradation curves at various initial concentrations; (b) the corresponding linearized plots of pseudo-second order adsorption kinetics; and (c) the corresponding linearized plots of pseudo-first order photodegradation kinetics.

might be due to the adsorption of insensitive species on the photocatalyst surface [65] and the supporting matrix [26]. Our observation was in good agreement with the report by Zhu et al. [3, 28, 29], who used a similar photocatalyst for the removal of Congo red, CI acid red 66, and methyl orange.

\section{Conclusion}

Chitosan encapsulated CdS catalyst was prepared and the catalyst showed effective degradation of methyl orange. The UV-visible absorption spectra indicated that the catalytic photodegradation process was more efficient for chromophores than for aromatic moiety of the dye molecules. The mechanism of dye degradation consists of physical adsorption, followed by a photochemical oxidation process. The adsorption process followed the pseudo-second order adsorption kinetics, while the photodegradation step followed the pseudo-first order kinetics. The effect of dosage, initial dye concentration, $\mathrm{pH}$, and cross-linking agents on dye removal and catalyst composite reusability were investigated. The results showed that the catalyst performed well at acidic $\mathrm{pH}(99 \%$ removal at $\mathrm{pH} 4)$ but 


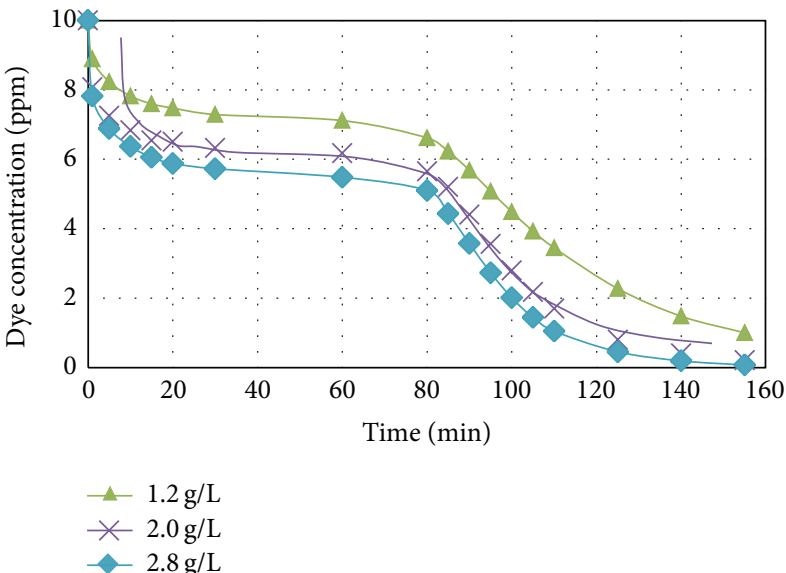

(a)

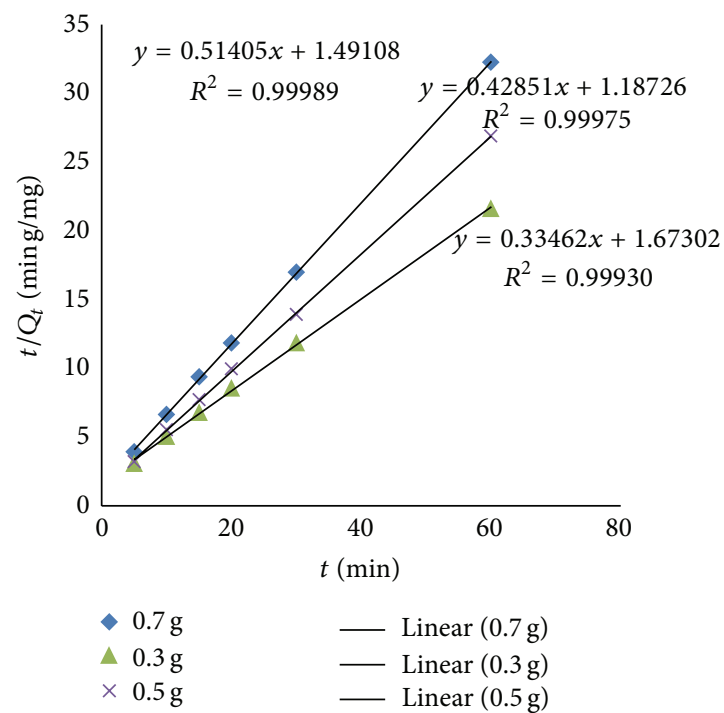

(b)

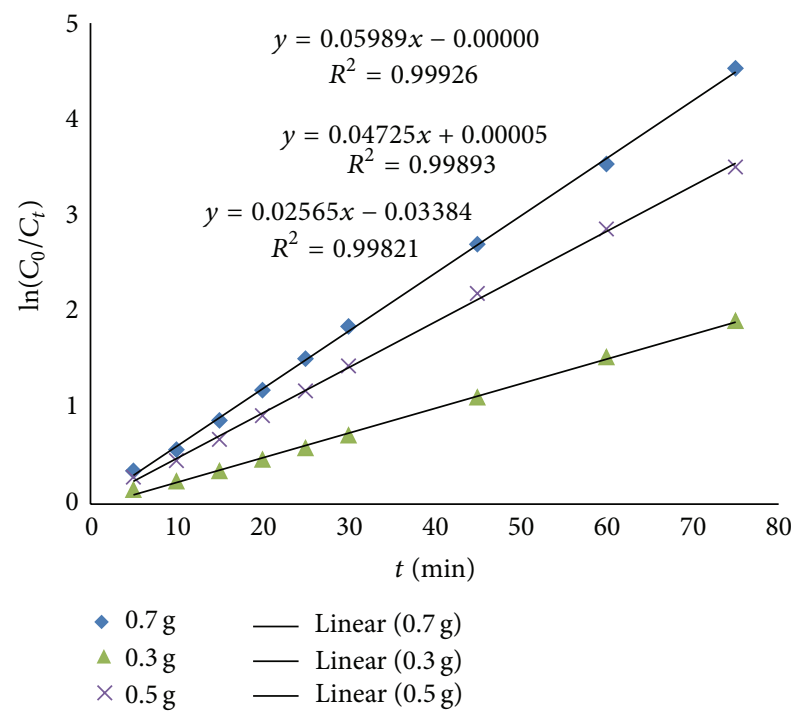

(c)

FIGURE 13: (a) Methyl orange adsorption-photodegradation curves by various dosages of photocatalyst composite; (b) the corresponding linearized plots of pseudo-second order adsorption kinetics; and (c) the corresponding linearized plots of pseudo-first order photodegradation kinetics.

poorly at alkaline $\mathrm{pH}$ (54\% removal at $\mathrm{pH} 10)$. Increasing the catalyst dosage could improve the removal efficiency, while increasing the initial dye concentration from 5 to $20 \mathrm{ppm}$ could decrease the dye removal efficiency with the kinetic rate constant $\left(k_{1 p}\right)$ decreasing from $0.55 \mathrm{~min}^{-1}$ to $0.12 \mathrm{~min}^{-1}$. The chitosan encapsulated CdS catalyst with and without epichlorohydrin cross-linking demonstrated the same $k_{1 p}$ of $0.026 \mathrm{~min}^{-1}$ and the same final dye removal of $90 \%$. Epichlorohydrin cross-linking caused almost no changes in dye removal characteristics. On the other hand, the glutaraldehyde cross-linking method enhanced adsorption but reduced photodegradation efficiency slightly. It is worth it to notice that the catalyst composite could be reused but the photocatalytic activity dropped successively in each cycle.

\section{Highlights}

Efficient decolorization of methyl orange solution by visible light induced photocatalysis using chitosan immobilized nanosize CdS. Dye removal using fluidized bed photoreactor loaded with cross-linked chitosan immobilized nanophotocatalyst. Cross-linked chitosan can help the recycle of the photocatalyst. 

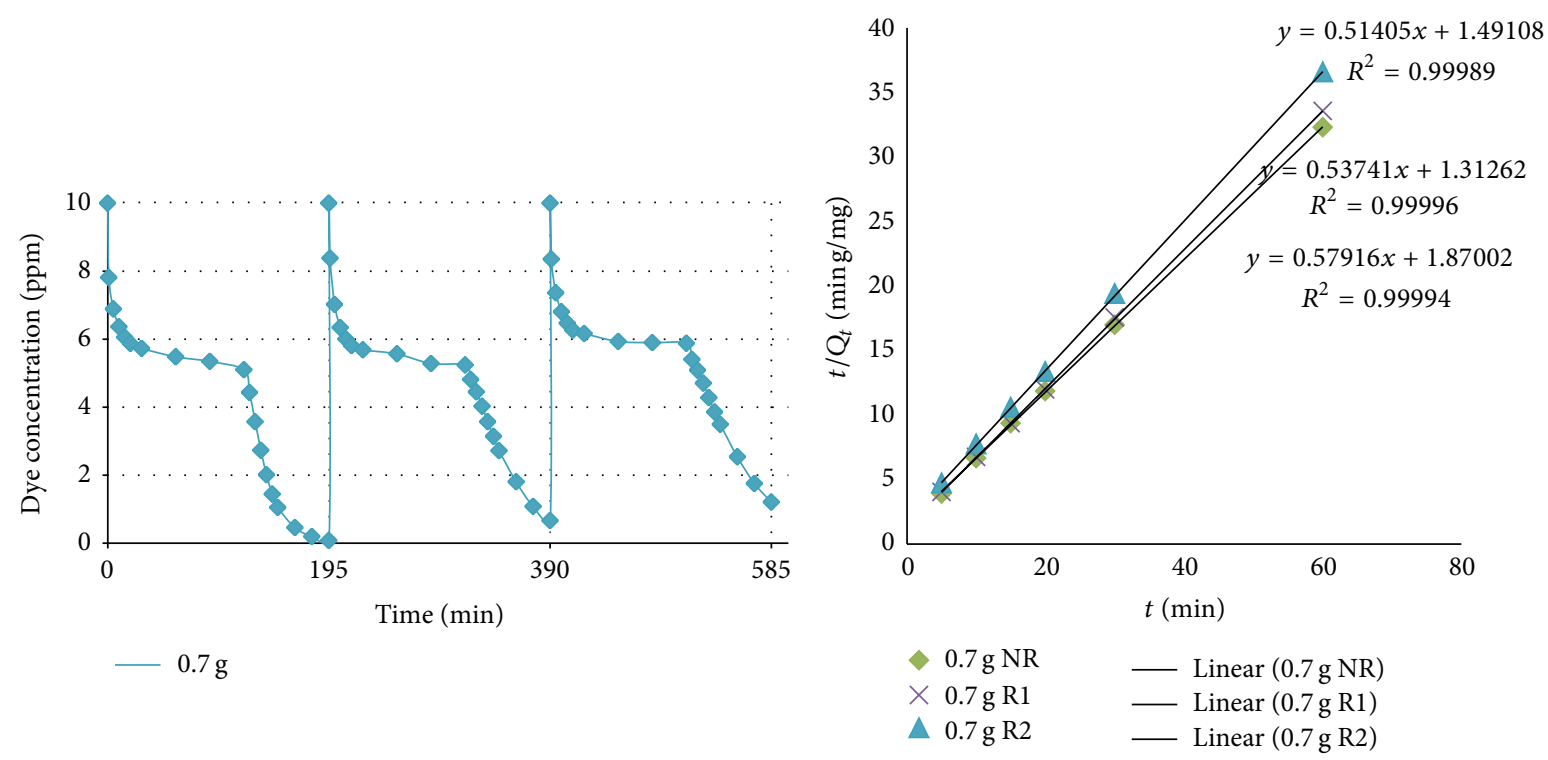

(a)

(b)

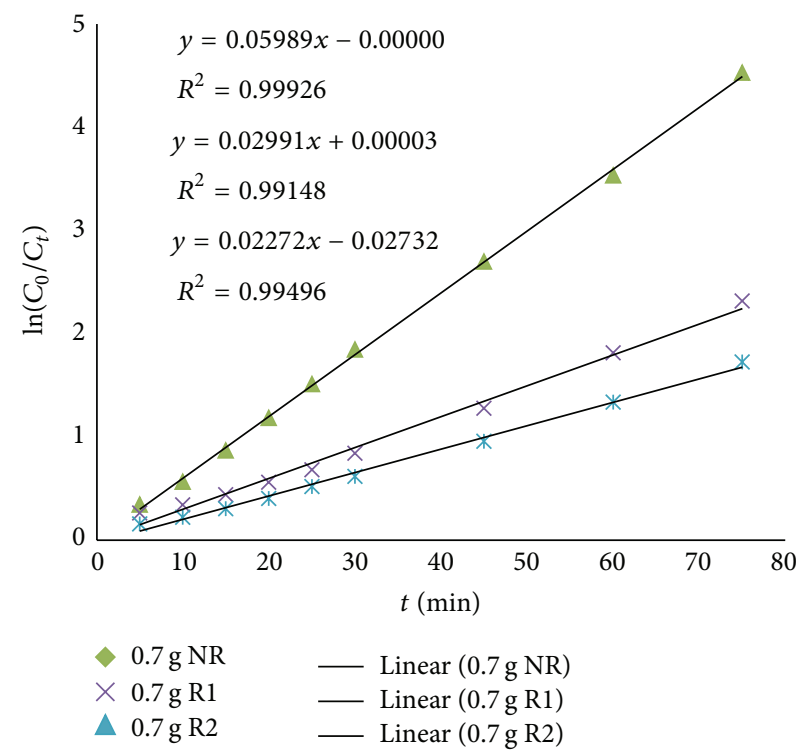

(c)

FIGURE 14: Multicycle MO adsorption-photodegradation curves of experiments with various dosages.

\section{Conflict of Interests}

The authors declare that there is no conflict of interests regarding the publication of this paper.

\section{Authors' Contribution}

Wai Szeto, Chi Wai Kan, and C. W. M. Yuen contributed equally to this paper.

\section{Acknowledgment}

The authors would like to acknowledge (i) the research Grant (Grant no. G-YM35) allocated to Department of Applied
Biology and Chemical Technology and (ii) the MPhil. Studentship allocated to Institute of Textiles and Clothing, both of which were provided by the Hong Kong Polytechnic University.

\section{References}

[1] G. M. Walker and L. R. Weatherley, "Fixed bed adsorption of acid dyes onto activated carbon," Environmental Pollution, vol. 99, no. 1, pp. 133-136, 1998.

[2] S. Sirianuntapiboon and J. Sansak, "Treatability studies with granular activated carbon (GAC) and sequencing batch reactor (SBR) system for textile wastewater containing direct dyes," Journal of Hazardous Materials, vol. 159, no. 2-3, pp. 404-411, 2008. 
[3] H. Y. Zhu, R. Jiang, L. Xiao et al., "Photocatalytic decolorization and degradation of Congo Red on innovative crosslinked chitosan/nano-CdS composite catalyst under visible light irradiation," Journal of Hazardous Materials, vol. 169, no. 1-3, pp. 933-940, 2009.

[4] Z. Zainal, L. K. Hui, M. Z. Hussein, A. H. Abdullah, and I. M. K. R. Hamadneh, "Characterization of $\mathrm{TiO}_{2}-$ Chitosan/Glass photocatalyst for the removal of a monoazo dye via photodegradation-adsorption process," Journal of Hazardous Materials, vol. 164, no. 1, pp. 138-145, 2009.

[5] S. Steffan, L. Bardi, and M. Marzona, "Azo dye biodegradation by microbial cultures immobilized in alginate beads," Environment International, vol. 31, no. 2, pp. 201-205, 2005.

[6] G. M. Walker and L. R. Weatherley, "Biodegradation and biosorption of acid anthraquinone dye," Environmental Pollution, vol. 108, no. 2, pp. 219-223, 2000.

[7] B. W. Manning, C. E. Cerniglia, and T. W. Federle, "Metabolism of the benzidine-based azo dye direct black 38 by human intestinal microbiota," Applied and Environmental Microbiology, vol. 50, no. 1, pp. 10-15, 1985.

[8] C. Karunakaran, R. Dhanalakshmi, P. Gomathisankar, and G. Manikandan, "Enhanced phenol-photodegradation by particulate semiconductor mixtures: interparticle electron-jump," Journal of Hazardous Materials, vol. 176, no. 1-3, pp. 799-806, 2010.

[9] H. Yan, W. Pan, D. A. Ping, Y. Jing, H. Y. Ping, and Y. Yong, "Preparation of CdS nanoparticles with reverse micelle method and photo-degradation of malachite green dye," Journal of Inorganic Materials, vol. 25, pp. 1221-1227, 2010.

[10] D. I. Petkowicz, S. B. C. Pergher, C. D. S. da Silva, Z. N. da Rocha, and J. H. Z. dos Santos, "Catalytic photodegradation of dyes by in situ zeolite-supported titania," Chemical Engineering Journal, vol. 158, no. 3, pp. 505-512, 2010.

[11] S. K. Papageorgiou, F. K. Katsaros, E. P. Favvas et al., "Alginate fibers as photocatalyst immobilizing agents applied in hybrid photocatalytic/ultrafiltration water treatment processes," Water Research, vol. 46, no. 6, pp. 1858-1872, 2012.

[12] A. G. S. Prado, E. A. Faria, J. R. Souzade, and J. D. Torres, "Ammonium complex of niobium as a precursor for the hydrothermal preparation of cellulose acetate $/ \mathrm{Nb}_{2} \mathrm{O}_{5}$ photocatalyst," Journal of Molecular Catalysis A: Chemical, vol. 237, no. 1-2, pp. 115-119, 2005.

[13] H. Y. Zhu, R. Jiang, Y. Q. Fu et al., "Effective photocatalytic decolorization of methyl orange utilizing $\mathrm{TiO}_{2} / \mathrm{ZnO} /$ chitosan nanocomposite films under simulated solar irradiation," Desalination, vol. 286, pp. 41-48, 2012.

[14] R. Afeesh, N. A. M. Barakat, S. S. Al-Deyab, A. Yousef, and H. Y. Kim, "Nematic shaped cadmium sulfide doped electrospun nanofiber mat: highly efficient, reusable, solar light photocatalyst," Colloids and Surfaces A: Physicochemical and Engineering Aspects, vol. 409, pp. 21-29, 2012.

[15] A. R. Unnithan, N. A. M. Barakat, M. F. Abadir, A. Yousef, and H. Y. Kim, "Novel CdPdS/PVAc core-shell nanofibers as an effective photocatalyst for organic pollutants degradation," Journal of Molecular Catalysis A: Chemical, vol. 363-364, pp. 186-194, 2012.

[16] A. H. Jawad and M. A. Nawi, "Oxidation of crosslinked chitosan-epichlorohydrine film and its application with $\mathrm{TiO}_{2}$ for phenol removal," Carbohydrate Polymers, vol. 90, no. 1, pp. 87-94, 2012.

[17] Y. Gao and H. T. Liu, "Preparation and catalytic property study of a novel kind of suspended photocatalyst of $\mathrm{TiO}_{2}$-activated carbon immobilized on silicone rubber film," Materials Chemistry and Physics, vol. 92, no. 2-3, pp. 604-608, 2005.

[18] H.-H. Tseng, W. W. Lee, M.-C. Wei, B.-S. Huang, M.-C. Hsieh, and P.-Y. Cheng, "Synthesis of $\mathrm{TiO}_{2} / \mathrm{SBA}-15$ photocatalyst for the azo dye decolorization through the polyol method," Chemical Engineering Journal, vol. 210, pp. 529-538, 2012.

[19] J. Chen, P. Zhou, J. Li, and Y. Wang, "Studies on the photocatalytic performance of cuprous oxide/chitosan nanocomposites activated by visible light," Carbohydrate Polymers, vol. 72, no. 1, pp. 128-132, 2008.

[20] C. H. Cao, L. Xiao, L. Liu, H. Y. Zhu, C. H. Chen, and L. Gao, "Visible-light photocatalytic decolorization of reactive brilliant red $\mathrm{X}-3 \mathrm{~B}$ on $\mathrm{Cu}_{2} \mathrm{O} /$ crosslinked-chitosan nanocomposites prepared via one step process," Applied Surface Science, vol. 271, pp. 105-112, 2013.

[21] Y. H. Yang, N. Ren, Y. H. Zhang, and Y. Tang, "Nanosized cadmium sulfide in polyelectrolyte protected mesoporous sphere: a stable and regeneratable photocatalyst for visible-light-induced removal of organic pollutants," Journal of Photochemistry and Photobiology A: Chemistry, vol. 201, no. 2-3, pp. 111-120, 2009.

[22] X. Tao, J. Su, L. Wang, and J. Chen, "A new heterogeneous catalytic system for wastewater treatment: Fe-immobilized polyelectrolyte microshells for accumulation and visible lightassisted photooxidative degradation of dye pollutants," Journal of Molecular Catalysis A: Chemical, vol. 280, no. 1-2, pp. 186-193, 2008.

[23] Y. M. Lee and W. J. Lee, "Degradation of trichloroethylene by $\mathrm{Fe}$ (II) chelated with cross-linked chitosan in a modified Fenton reaction," Journal of Hazardous Materials, vol. 178, no. 1-, pp. 187-193, 2010.

[24] R. Šuláková, R. Hrdina, and G. M. B. Soares, "Oxidation of azo textile soluble dyes with hydrogen peroxide in the presence of $\mathrm{Cu}(\mathrm{II})$-chitosan heterogeneous catalysts," Dyes and Pigments, vol. 73, no. 1, pp. 19-24, 2007.

[25] M. A. Nawi, S. Sabar, A. H. Jawad, and W. S. W. Ngah, "Adsorption of Reactive Red 4 by immobilized chitosan on glass plates: towards the design of immobilized $\mathrm{TiO}_{2}$-chitosan synergistic photocatalyst-adsorption bilayer system," Biochemical Engineering Journal, vol. 49, no. 3, pp. 317-325, 2010.

[26] H. Y. Zhu, L. Xiao, R. Jiang, G. M. Zeng, and L. Liu, "Efficient decolorization of azo dye solution by visible light-induced photocatalytic process using $\mathrm{SnO}_{2} / \mathrm{ZnO}$ heterojunction immobilized in chitosan matrix," Chemical Engineering Journal, vol. 172, no. 2-3, pp. 746-753, 2011.

[27] Q. Li, H. Su, and T. Tan, "Synthesis of ion-imprinted chitosan$\mathrm{TiO}_{2}$ adsorbent and its multi-functional performances," Biochemical Engineering Journal, vol. 38, no. 2, pp. 212-218, 2008.

[28] R. Jiang, H. Y. Zhu, X. D. Li, and L. Xiao, "Visible light photocatalytic decolourization of C. I. Acid Red 66 by chitosan capped CdS composite nanoparticles," Chemical Engineering Journal, vol. 152, no. 2-3, pp. 537-542, 2009.

[29] R. Jiang, H. Zhu, and X. Li, "Adsorption and visible light photocatalytic decolorization of methyl orange using innovative chitosan capped CdS composite," in Proceedings of the 3 rd International Conference on Bioinformatics and Biomedical Engineering, iCBBE 2009), 4, p. 1, June 2009.

[30] W. Nam, K. Woo, and G. Han, "Photooxidation of anionic surfactant (sodium lauryl sulfate) in a three-phase fluidized bed reactor using $\mathrm{TiO}_{2} / \mathrm{SiO}_{2}$ photocatalyst," Journal of Industrial and Engineering Chemistry, vol. 15, no. 3, pp. 348-353, 2009.

[31] Y. Kang, K. J. Woo, M. H. Ko, and S. D. Kim, "Particle dispersion and pressure fluctuations in three-phase fluidized 
beds," Chemical Engineering Science, vol. 52, no. 21-22, pp. 37233732, 1997.

[32] Y. J. Cho, S. J. Kim, S. H. Nam, Y. Kang, and S. D. Kim, "Heat transfer and bubble properties in three-phase circulating fluidized beds," Chemical Engineering Science, vol. 56, no. 21-22, pp. 6107-6115, 2001.

[33] W. S. Wan Ngah, C. S. Endud, and R. Mayanar, "Removal of copper(II) ions from aqueous solution onto chitosan and crosslinked chitosan beads," Reactive \& Functional Polymers, vol. 50, no. 2, pp. 181-190, 2002.

[34] M. I. El-Khaiary, G. F. Malash, and Y. Ho, "On the use of linearized pseudo-second-order kinetic equations for modeling adsorption systems," Desalination, vol. 257, no. 1-3, pp. 93-101, 2010.

[35] J. Bandara, K. Tennakone, and P. P. B. Jayatilaka, "Composite tin and zinc oxide nanocrystalline particles for enhanced charge separation in sensitized degradation of dyes," Chemosphere, vol. 49, no. 4, pp. 439-445, 2002.

[36] T. Chen, Y. Zheng, J. Lin, and G. Chen, "Study on the photocatalytic degradation of methyl orange in water using $\mathrm{Ag} / \mathrm{ZnO}$ as catalyst by liquid chromatography electrospray ionization iontrap mass spectrometry," Journal of the American Society for Mass Spectrometry, vol. 19, no. 7, pp. 997-1003, 2008.

[37] K. Hayat, M. A. Gondal, M. M. Khaled, Z. H. Yamani, and S. Ahmed, "Laser induced photocatalytic degradation of hazardous dye (Safranin-O) using self synthesized nanocrystalline $\mathrm{WO}_{3}$, Journal of Hazardous Materials, vol. 186, no. 2-3, pp. 1226-1233, 2011.

[38] W. Z. Yin, W. Z. Wang, L. Zhou, S. M. Sun, and L. Zhang, "CTAB-assisted synthesis of monoclinic $\mathrm{BiVO}_{4}$ photocatalyst and its highly efficient degradation of organic dye under visiblelight irradiation," Journal of Hazardous Materials, vol. 173, no. 1-3, pp. 194-199, 2010.

[39] N. Soltani, E. Gharibshahi, and E. Saion, "Band gap of cubic and hexagonal cds quantum dots-experimental and theoretical studies," Chalcogenide Letters, vol. 9, no. 7, pp. 321-328, 2012.

[40] P. Reyes and S. Velumani, "Structural and optical characterization of mechanochemically synthesized copper doped CdS nanopowders," Materials Science and Engineering B, vol. 177, no. 16, pp. 1452-1459, 2012.

[41] E. Maier, A. Fischereder, W. Haas et al., "Metal sulfide-polymer nanocomposite thin films prepared by a direct formation route for photovoltaic applications," Thin Solid Films, vol. 519, no. 13, pp. 4201-4206, 2011.

[42] R. Jiang, H. Y. Zhu, J. Yao, Y. Q. Fu, and Y. J. Guan, "Chitosan hydrogel films as a template for mild biosynthesis of CdS quantum dots with highly efficient photocatalytic activity," Applied Surface Science, vol. 258, no. 8, pp. 3513-3518, 2012.

[43] F. Tasselli, A. Mirmohseni, M. S. Seyed Dorraji, and A. Figoli, "Mechanical, swelling and adsorptive properties of dry-wet spun chitosan hollow fibers crosslinked with glutaraldehyde," Reactive \& Functional Polymers, vol. 73, no. 1, pp. 218-223, 2013.

[44] O. A. C. Jr. Monteiro and C. Airoldi, "Some studies of crosslinking chitosan-glutaraldehyde interaction in a homogeneous system," International Journal of Biological Macromolecules, vol. 26, no. 2-3, pp. 119-128, 1999.

[45] S. Margel and A. Rembaum, "Synthesis and characterization of poly(glutaraldehyde). A potential reagent for protein immobilization and cell separation," Macromolecules, vol. 13, no. 1, pp. 19-24, 1980.

[46] H. W. Hu, J. H. Xin, A. Chan, and L. He, "Glutaraldehydechitosan and poly (vinyl alcohol) blends, and fluorescence of their nano-silica composite films," Carbohydrate Polymers, vol. 91, no. 1, pp. 305-313, 2013.

[47] W. S. W. Ngah and S. Fatinathan, "Pb(II) biosorption using chitosan and chitosan derivatives beads: equilibrium, ion exchange and mechanism studies," Journal of Environmental Sciences, vol. 22, no. 3, pp. 338-346, 2010.

[48] M. M. Beppu, R. S. Vieira, C. G. Aimoli, and C. C. Santana, "Crosslinking of chitosan membranes using glutaraldehyde: effect on ion permeability and water absorption," Journal of Membrane Science, vol. 301, no. 1-2, pp. 126-130, 2007.

[49] V. Kumar, S. Kr. Sharma, T. P. Sharma, and V. Singh, "Band gap determination in thick films from reflectance measurements," Optical Materials, vol. 12, no. 1, pp. 115-119, 1999.

[50] G. P. Joshi, N. S. Saxena, R. Mangal, A. Mishra, and T. P. Sharma, "Band gap determination of Ni-Zn ferrites," Bulletin of Materials Science, vol. 26, no. 4, pp. 387-389, 2003.

[51] X. Yong and M. A. A. Schoonen, "The absolute energy positions of conduction and valence bands of selected semiconducting minerals," American Mineralogist, vol. 85, no. 3-4, pp. 543-556, 2000.

[52] R. Laus, T. G. Costa, B. Szpoganicz, and V. T. Fávere, "Adsorption and desorption of $\mathrm{Cu}(\mathrm{II}), \mathrm{Cd}(\mathrm{II})$ and $\mathrm{Pb}(\mathrm{II})$ ions using chitosan crosslinked with epichlorohydrin-triphosphate as the adsorbent," Journal of Hazardous Materials, vol. 183, no. 1-3, pp. 233-241, 2010.

[53] W. S. W. Ngah and S. Fatinathan, "Chitosan flakes and chitosanGLA beads for adsorption of p-nitrophenol in aqueous solution," Colloids and Surfaces A: Physicochemical and Engineering Aspects, vol. 277, no. 1-3, pp. 214-222, 2006.

[54] I. El Saliby, M. Shahid, A. McDonagh, H. K. Shon, and J. Kim, "Photodesorption of organic matter from titanium dioxide particles in aqueous media," Journal of Industrial and Engineering Chemistry, vol. 18, no. 5, pp. 1774-1780, 2012.

[55] R. B. Bjorklund, S. Karlsson, H. Borén, B. Allard, and I. Lundström, "Photodesorption of fulvic acid from iron oxide surfaces into aqueous solutions," Applied Surface Science, vol. 174, no. 2, pp. 166-175, 2001.

[56] H. K. Shon, S. Vigneswaran, H. H. Ngo, and J.-H. Kim, "Chemical coupling of photocatalysis with flocculation and adsorption in the removal of organic matter," Water Research, vol. 39, no. 12, pp. 2549-2558, 2005.

[57] L. Erdei, N. Arecrachakul, and S. Vigneswaran, "A combined photocatalytic slurry reactor-immersed membrane module system for advanced wastewater treatment," Separation and Purification Technology, vol. 62, no. 2, pp. 382-388, 2008.

[58] Z. Ambrus, K. Mogyorósi, Á. Szalai et al., "Low temperature synthesis, characterization and substrate-dependent photocatalytic activity of nanocrystalline $\mathrm{TiO}_{2}$ with tailor-made rutile to anatase ratio," Applied Catalysis A: General, vol. 340, no. 2, pp. 153-161, 2008.

[59] A. H. Zyoud, N. Zaatar, I. Saadeddin et al., "CdS-sensitized $\mathrm{TiO}_{2}$ in phenazopyridine photo-degradation: catalyst efficiency, stability and feasibility assessment," Journal of Hazardous Materials, vol. 173, no. 1-3, pp. 318-325, 2010.

[60] S. Qourzal, N. Barka, M. Tamimi, A. Assabbane, and Y. AitIchou, "Photodegradation of 2-naphthol in water by artificial light illumination using $\mathrm{TiO}_{2}$ photocatalyst: Identification of intermediates and the reaction pathway," Applied Catalysis A: General, vol. 334, no. 1-2, pp. 386-393, 2008.

[61] H. R. Pouretedal and A. Kadkhodaie, "Synthetic $\mathrm{CeO}_{2}$ nanoparticle catalysis of methylene blue photodegradation: kinetics and 
mechanism," Cuihua Xuebao/Chinese Journal of Catalysis, vol. 31, no. 11, pp. 1328-1334, 2010.

[62] M. A. Nawi and S. Sabar, "Photocatalytic decolourisation of Reactive Red 4 dye by an immobilised $\mathrm{TiO}_{2}$ /chitosan layer by layer system," Journal of Colloid and Interface Science, vol. 372, no. 1, pp. 80-87, 2012.

[63] F. Shahrezaei, Y. Mansouri, A. A. L. Zinatizadeh, and A. Akhbari, "Process modeling and kinetic evaluation of petroleum refinery wastewater treatment in a photocatalytic reactor using $\mathrm{TiO}_{2}$ nanoparticles," Powder Technology, vol. 221, pp. 203-212, 2012.

[64] M. Pelaez, A. A. de la Cruz, K. O'Shea, P. Falaras, and D. D. Dionysiou, "Effects of water parameters on the degradation of microcystin-LR under visible light-activated $\mathrm{TiO}_{2}$ photocatalyst," Water Research, vol. 45, no. 12, pp. 3787-3796, 2011.

[65] S. Song, L. Xu, Z. He et al., "Photocatalytic degradation of C.I. Direct Red 23 in aqueous solutions under UV irradiation using $\mathrm{SrTiO}_{3} / \mathrm{CeO}_{2}$ composite as the catalyst," Journal of Hazardous Materials, vol. 152, no. 3, pp. 1301-1308, 2008.

[66] S. F. Chen and Y. Z. Liu, "Study on the photocatalytic degradation of glyphosate by $\mathrm{TiO}_{2}$ photocatalyst," Chemosphere, vol. 67, no. 5, pp. 1010-1017, 2007.

[67] N. M. Mahmoodi, M. Arami, and J. Zhang, "Preparation and photocatalytic activity of immobilized composite photocatalyst (titania nanoparticle/activated carbon)," Journal of Alloys and Compounds, vol. 509, no. 14, pp. 4754-4764, 2011.

[68] A. F. Caliman, C. Cojocaru, A. Antoniadis, and I. Poulios, "Optimized photocatalytic degradation of Alcian Blue 8 GX in the presence of $\mathrm{TiO}_{2}$ suspensions," Journal of Hazardous Materials, vol. 144, no. 1-2, pp. 265-273, 2007.

[69] R. S. Vieira and M. M. Beppu, "Interaction of natural and crosslinked chitosan membranes with $\mathrm{Hg}(\mathrm{II})$ ions," Colloids and Surfaces A: Physicochemical and Engineering Aspects, vol. 279, no. 1-3, pp. 196-207, 2006.

[70] H. Qiu, L. Lv, B. Pan, Q. Zhang, and W. Zhang, "Critical review in adsorption kinetic models," Journal of Zhejiang University: Science A, vol. 10, no. 5, pp. 716-724, 2009. 

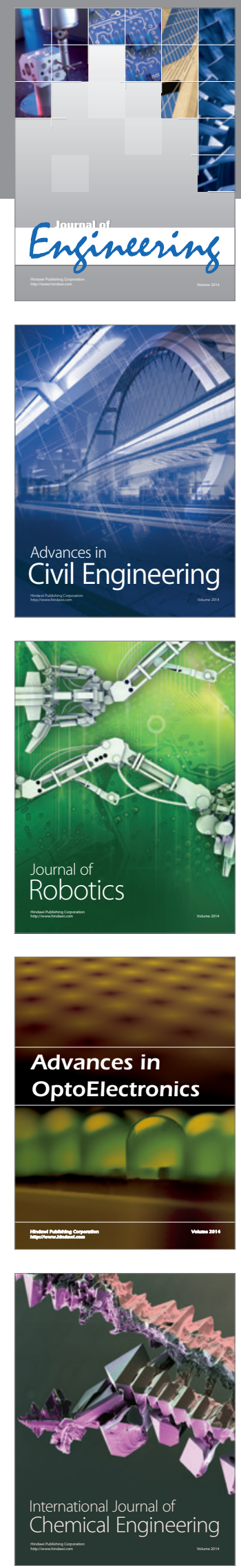

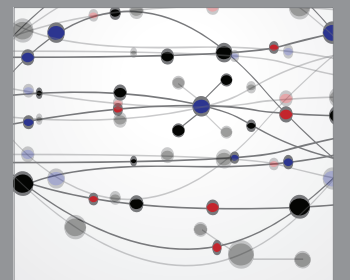

The Scientific World Journal
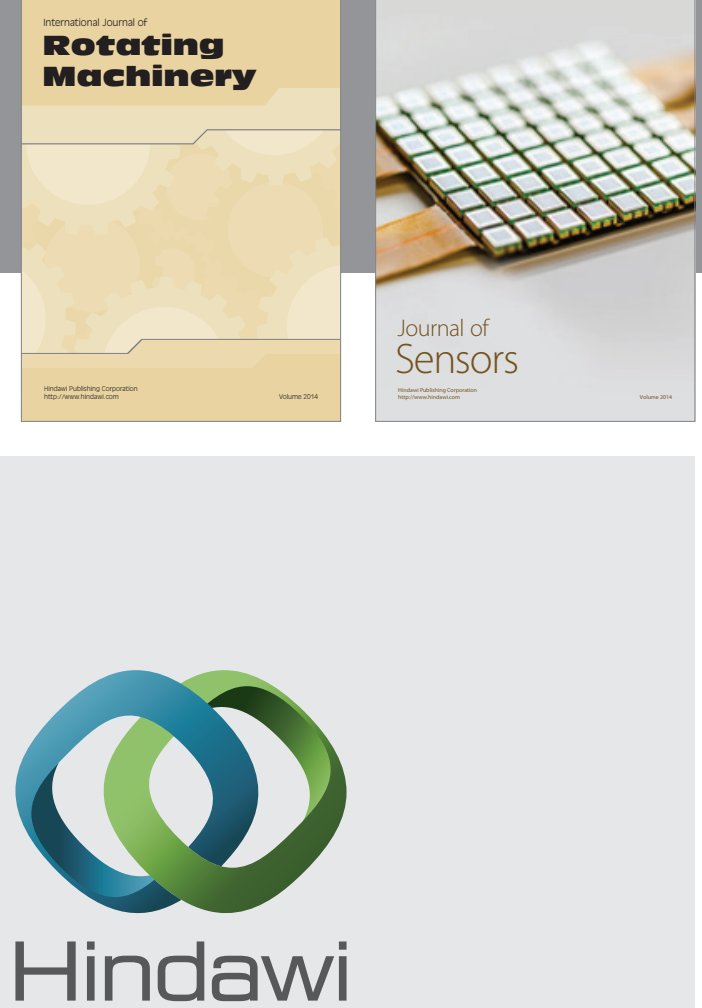

Submit your manuscripts at http://www.hindawi.com
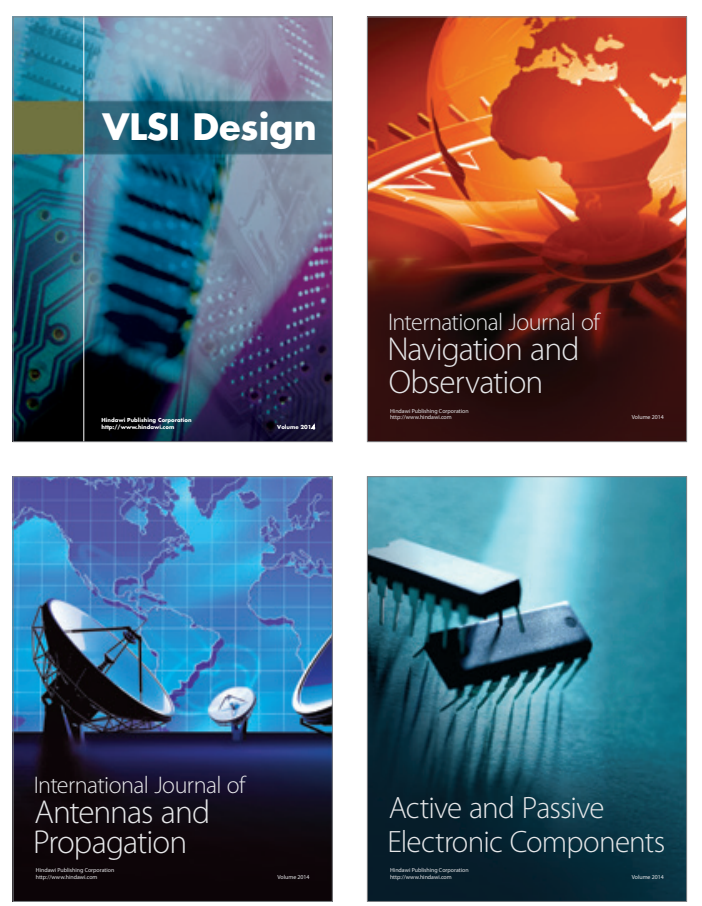
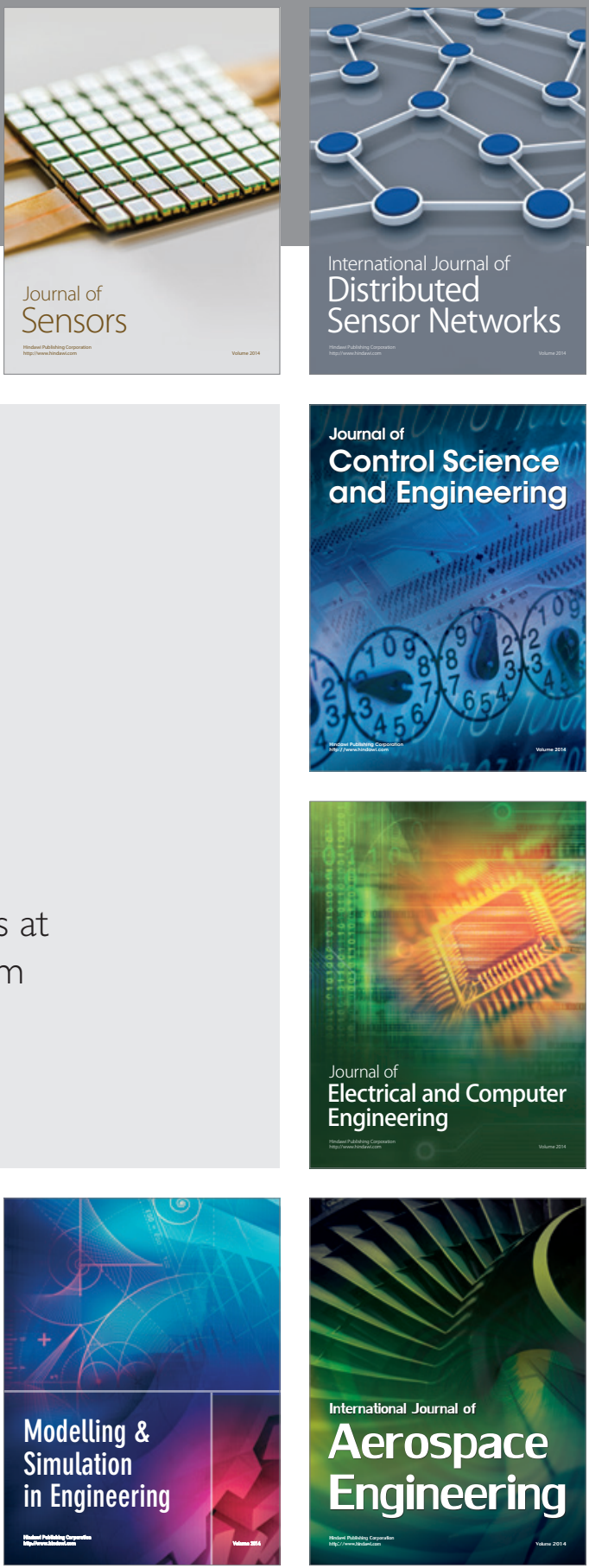

Journal of

Control Science

and Engineering
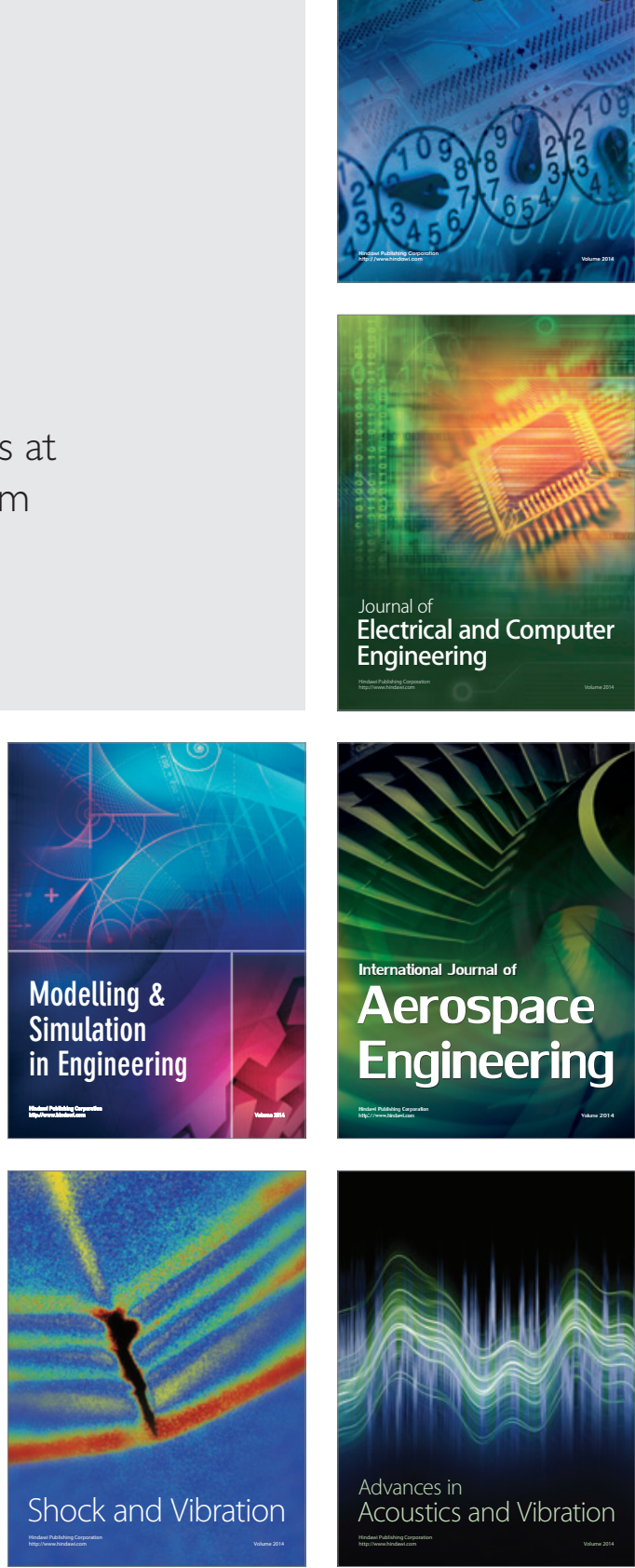